\title{
Relaxor-to-ferroelectric crossover and disruption of polar order in 'empty' tetragonal tungsten bronzes
}

\author{
Jonathan Gardner ${ }^{1}$, Fengjiao $\mathrm{Yu}^{1}$, Chiu Tang ${ }^{2}$, Winfried Kockelmann ${ }^{3}$, Wuzong Zhou ${ }^{1}$, \\ Finlay D. Morrison ${ }^{1, *}$ \\ ${ }^{1}$ EaStCHEM School of Chemistry, University of St Andrews, North Haugh, St Andrews, \\ KY16 9ST, UK \\ ${ }^{2}$ Diamond Light Source Ltd., Harwell Science and Innovation Campus, Didcot OX11 0DE, \\ UK \\ ${ }^{3}$ ISIS Facility, Rutherford Appleton Laboratory, Chilton, Didcot OX11 0QX, UK
}

\begin{abstract}
Combined temperature-dependent structural and electrical characterization of a series of "empty" ferroelectric tetragonal tungsten bronzes (TTBs) of composition $\mathrm{Ba}_{4}\left(\mathrm{La}_{1-}\right.$ $\left.{ }_{x} \mathrm{Nd}_{\mathrm{x}}\right)_{0.67} \square_{1.33} \mathrm{Nb}_{10} \mathrm{O}_{30}$ are reported. The La-material exhibits a temperature dependent crossover from relaxor-ferroelectric to polar (but non-ferroelectric) to linear dielectric behavior. The loss of ferroelectric switching in the polar, non-ferroelectric phase is accompanied by disorder associated with structural relaxation due the significant vacancy concentration at the A1-perovskite-like site. In this disordered regime, large polarization can be re-established with application of sufficient electric field, however relaxation back into the disordered phase occurs on removal of the field as indicated by the loss of remenant polarization. The field against which "backswitching" (depolarization) occurs increases with temperature indicating increasing stability of the disordered regime. The disordered phase can be de-stabilized by substituting $\mathrm{Nd}$ for $\mathrm{La}$ at the A1-site and which reintroduces "normal" ferroelectric behavior.
\end{abstract}

\section{Introduction}

The field of polar dielectrics (piezo-, pyro- and ferro-electrics) is dominated by oxides with the perovskite structure. ${ }^{1,2}$ The role of compositional tuning of perovskites and structural consequences which control the physical properties have been intensively studied and are relatively well understood. Ferroelectric and relaxor perovskites are particularly prevalent and the origins of the 'crossover' from normal ferroelectric to relaxor behavior in these compounds have been widely studied. Relaxor behavior is associated with local disorder, which in perovskites is most commonly associated with mixed cations on either the A-site, e.g. $\mathrm{Pb}_{1-\mathrm{x}} \mathrm{La}_{2 \mathrm{x} / 3}(\mathrm{Zr}, \mathrm{Ti}) \mathrm{O}_{3}(\mathrm{PLZT}),{ }^{3}$ or B-site e.g. $\mathrm{PbMg}_{1 / 3} \mathrm{Nb}_{2 / 3} \mathrm{O}_{3}(\mathrm{PMN})^{4}$ or $\mathrm{PbSc}_{1 / 2} \mathrm{Ta}_{1 / 2} \mathrm{O}_{3}$ (PST). ${ }^{5,6}$ PST demonstrates the influence of disorder very succinctly: when the $\mathrm{Sc}^{3+}$ and $\mathrm{Ta}^{5+}$ cations are randomly distributed (disordered) relaxor behavior results, but when the cations are ordered, a normal $1^{\text {st }}$ order ferroelectric transition is observed. ${ }^{6}$ The defining qualitative 
properties of relaxors (with respect to normal ferroelectrics) are the differing polarization relaxation dynamics as a result of disorder. These differences are manifested as a large temperature- and frequency-dependent dispersion in both the real and imaginary components of the complex permittivity. The properties of relaxor and relaxor ferroelectric (RFE) systems have been subject to a number of reviews. ${ }^{2,-11}$ While perovskite relaxors are dominant, relaxor-like behavior has been reported in other perovskite-related structure types such as the Aurivillius, $\mathrm{Bi}_{2} \mathrm{O}_{2}{ }^{2-}$-layered structures and tetragonal tungsten bronzes, but these systems are less widely studied.

The tetragonal tungsten bronze oxides (TTBs), general formula $\mathrm{A} 1_{2} \mathrm{~A} 2{ }_{4} \mathrm{~B} 1_{2} \mathrm{~B} 2{ }_{8} \mathrm{C}_{4} \mathrm{O}_{30}$, contain perovskite-like units which are inter-linked by an oxide octahedron to form channels ${ }^{12}$ with triangular, quadrilateral and pentagonal cross-sections, Figure 1. This relatively open TTB structure can accommodate a wide array of cations resulting in a rich diversity of compositions with this structure type. Variable occupancy of the A- and C-sites allows additional compositional degrees of freedom and therefore structural complexity. When $\mathrm{C}$ is unoccupied the structure may be described as 'filled' e.g. $\mathrm{Ba}_{4} \mathrm{Na}_{2} \mathrm{Nb}_{10} \mathrm{O}_{30}(\mathrm{BNN})^{12}$ or 'unfilled' e.g. $(\mathrm{Sr}, \mathrm{Ba})_{5} \mathrm{Nb}_{10} \mathrm{O}_{30}(\mathrm{SBN}),{ }^{13,14}$ contingent on whether vacancies are present in the A-sites. The less commonly reported 'stuffed' compounds have fully occupied A- and C-sites e.g. $\mathrm{K}_{6} \mathrm{Li}_{4} \mathrm{Nb}_{10} \mathrm{O}_{30}(\mathrm{KLN}){ }^{15}$

The TTB structural aristotype is tetragonal with crystallographic point group 4/mmm (typically with space group $P 4 / \mathrm{mbm}$ ) and has approximate cell dimensions of $a_{T T B}=b_{T T B} \approx$ $12.5 \AA$ and $c_{T T B} \approx 4 \AA \AA^{16}$ The simplest ferroelectric TTB is tetragonal with similar cell dimensions to the aristotype structure but with non-centrosymmetric symmetry, point group $4 \mathrm{~mm}$, with $\mathrm{P} 4 \mathrm{bm}$ the most commonly assigned space group. ${ }^{17}$ However, orthorhombic distortions of the structure are common, typically resulting in larger supercells. The simplest such orthorhombic superstructure requires a $c a .45^{\circ}$ rotation and $\sqrt{2}$ expansion of the unit cell in the $a b$ plane such that $a \approx b \approx \sqrt{ } 2 a_{T T B}$; such expansions can frequently occur in conjunction with doubling in the $c$-axis resulting in a further expansion of the superstructure. Such orthorhombic distortions are comparatively subtle, likely due to the relative rigidity of the TTB structure. ${ }^{18}$ Further doubling in $a$ and $b$ may lead to even larger superstructures, e.g. $\mathrm{Sr}_{4} \mathrm{Na}_{2} \mathrm{Nb}_{10} \mathrm{O}_{30}(\mathrm{SNN}), 2 \sqrt{2} a_{T T B} \times 2 \sqrt{2} b_{T T B} \times 2 c_{T T B} .{ }^{19}$ The origins of these superstructures are principally variations in the oxygen positions due to octahedral tilting out of the $a b$ plane..$^{20-}$ ${ }^{30}$. When these variations are periodic, i.e. modulated, and hence can be described by wave vectors the resulting structures can either be described in reciprocal as: commensurate when the wave vectors describing the modulation are some simple integers of the wave vectors of the original unit cell, and so can be described by a supercell; or incommensurate if the modulation wave vector is not translatable to a simple integer of the original unit cell wave vector. In the latter case, the structure may be described by a combination of the average unit cell together with the appropriate incommensurate wave vectors. If the positional variations are not periodic, then the material is disordered and no additional supercell may be assigned.

These structural variations, which can be considered as degrees of disorder on varying lengths-scales, have been attributed to be the origin of relaxor behavior in TTBs. ${ }^{20,21,23,29,31-}$ ${ }^{37}$ Levin and co-workers have linked particular superstructure types to relaxor $v s$. ferroelectric 
properties to the $\mathrm{M}$ cation size at the A1-site in the family of filled TTBs, $\mathrm{Ba}_{4} \mathrm{M}_{2} \mathrm{Ti}_{4} \mathrm{Nb}_{6} \mathrm{O}_{30}$. Smaller cations produce a commensurate $\sqrt{2} a_{T T B} \times \sqrt{2} b_{T T B} \times 2 c_{T T B}$ superstructure (space group Ima 2 ) and a normal ferroelectric response. ${ }^{21}$ For the radius of $\mathrm{M} \geq 1.36 \AA$ (La and $\mathrm{Bi}$ ) the compounds have incommensurately modulated superstructures with an approximate $\sqrt{2} a_{T T B} \times$ $2 \sqrt{2} b_{T T B} \times 2 c_{T T B}$ unit cell with space group $A m a 2$, and exhibit relaxor behavior. ${ }^{21}$ This latter structure had previously been reported by Bursill and Lin as one of two competing superstructures within $\mathrm{SBN}^{24}$ and by Labbé and co-workers as an approximation of the ambient temperature quasi-commensurate phase of BNN ${ }^{38}$ In their study Labbé et al. used an alternative orthorhombic setting Bbm2 with $a \approx 2 \sqrt{ } 2 a_{T T B}, b \approx \sqrt{ } 2 a_{T T B}$ and $c=2 c_{T T B}$ (which is a non-standard setting of $A m a 2$ where $a \approx \sqrt{ } 2 a_{T \text { TRB }}, b \approx 2 \sqrt{ } 2 b_{\text {TTB }}$ and $\left.c \approx 2 c_{T T B}\right){ }^{38}$

A recently proposed crystal-chemical framework predicts the influence of such structural modulations and the onset of relaxor or ferroelectric behavior. ${ }^{20}$ The model is based on the balance of two competing effects: large average A-cation size extends the $\mathrm{BO}_{6}$ octahedral in the $c$-axis enhancing polarizability of the $d^{0}$ cation favoring ferroelectric ordering but with incommensurate superstructure due to a modulated tilt pattern. Smaller relative A1-cations, denoted by a smaller tolerance factor of the perovskite "A1B2 $\mathrm{O}_{3}$ " sub-unit, results in larger (commensurate) octahedral tilting and long range polar ordering. In the absence of a strong driving force for polar ordering in the intermediate regime neither dominates resulting in a frustrated, incommensurate tilt system. These local structural modulations cause relaxor behavior. It is important to note that Chen and co-workers also proposed a model to predict relaxor vs. ferroelectric behavior but which considered only the difference in A-site cation radii. $^{37,39}$ As part of the proposed crystal-chemical framework ${ }^{20}$ and in earlier ${ }^{40}$ studies we $^{2}$ reported the properties of a family of "empty" TTBs of general formula TTBs $\mathrm{Ba}_{4} \mathrm{R}_{0.67} \square$ ${ }_{1.33} \mathrm{Nb}_{10} \mathrm{O}_{30}(\mathrm{R}=\mathrm{La}, \mathrm{Nd}, \mathrm{Sm}, \mathrm{Gd}$, Dy and $\mathrm{Y}$, and $\square=$ vacancy) and where the $\mathrm{Ba}$ and $\mathrm{R}$ solely occupy the A2 and A1 site, respectively. ${ }^{40}$ We use the term "empty" to denote TTBs where the A1 site is less than 50\% occupied to distinguish these compositions from the already widely studied unfilled compositions based on SBN. These empty TTB compounds fit within the crystal-chemical model with $\mathrm{R}=\mathrm{Nd}, \mathrm{Sm}, \mathrm{Gd}$, Dy and $\mathrm{Y}$ demonstrating normal ferroelectric behavior and the larger $\mathrm{R}=\mathrm{La}$ displays relaxor-like properties. However, the latter composition exhibits more complex behavior than that of the closely related filled analogue, $\mathrm{Ba}_{4} \mathrm{La}_{2} \mathrm{Ti}_{4} \mathrm{Nb}_{6} \mathrm{O}_{30},{ }^{21,}{ }^{40}$ in that on heating it displays an intermediate disordered polar phase before transforming to the high temperature paraelectric phase. In this contribution we report a more detailed structural investigation of $\mathrm{Ba}_{4} \mathrm{R}_{0.67} \square_{1.33} \mathrm{Nb}_{10} \mathrm{O}_{30}, \mathrm{R}=$ $\mathrm{La}$ and $\mathrm{Nd}$, using variable temperature high resolution neutron, synchrotron $\mathrm{x}$-ray and selected area electron diffraction in an attempt to link structural changes with the observed dielectric and polarization-field (P-E) response. We probe the sensitivity of the crossover from normal to relaxor ferroelectric by controlling the A1-cation size with the series $\mathrm{Ba}_{4}\left(\mathrm{La}_{1}\right.$ $\left.{ }_{x} \mathrm{Nd}_{\mathrm{x}}\right)_{0.67} \square_{1.33} \mathrm{Nb}_{10} \mathrm{O}_{30}$. Ferroelectric ordering is destabilized with increasing cation size (decreasing $x$ ) and allows the relaxor-ferroelectric crossover to be examined in detail. We discuss the role of disorder, most likely due to variations in oxygen positions, on disruption on long range polar ordering and the stability of such disorder as a function of temperature and electric field. 


\section{Experimental}

All samples were prepared using a conventional solid-state method. Precursor reagents, $\mathrm{BaCO}_{3}, \mathrm{Nb}_{2} \mathrm{O}_{5}, \mathrm{La}_{2} \mathrm{O}_{3}$, and $\mathrm{Nd}_{2} \mathrm{O}_{3}$ (Aldrich, all $>99 \%$ purity), were dried immediately prior to use. Powder mixtures of reagents, of appropriate stoichiometry to yield compositions $\mathrm{Ba}_{4}\left(\mathrm{La}_{1-\mathrm{x}} \mathrm{Nd}_{x}\right)_{0.67} \mathrm{Nb}_{10} \mathrm{O}_{30}(x=0,0.25,0.5,0.75$, and 1$)$, were homogenized with mortar and pestle then formed into loosely compacted bodies using a die press. An initial thermal treatment was carried out to calcine powder and initiate reaction; compounds were heated at $10{ }^{\circ} \mathrm{C} \mathrm{min}{ }^{-1}$ in a platinum lined alumina boat at $1000{ }^{\circ} \mathrm{C}$ for 2 hours, $1250{ }^{\circ} \mathrm{C}$ for 15 hours, then air quenched from $1000{ }^{\circ} \mathrm{C}$. Compounds were powdered, milled in ethanol using a planetary ball milled (1 hour, 600 r.p.m.), then uniaxially pressed into pellets of $0.5-2 \mathrm{~mm}$ thickness. Dense ( $>95 \%$ theoretical density) pellets for structural and electrical characterization were obtained by sintered at 1350 to $1400{ }^{\circ} \mathrm{C}$ for 6 hours $(x=0$ and 1$)$ or 12 hours $(x=0.25,0.5$, and 0.75$)$ with heating/cooling rates of $10{ }^{\circ} \mathrm{C} \mathrm{min}{ }^{-1}$.

High resolution powder x-ray diffraction (PXRD) was performed using synchrotron radiation $(\lambda=0.8269 \AA)$ on the I11 beamline at the Diamond Light Source synchrotron facility (Oxfordshire, UK). ${ }^{41}$ Sintered pellets were crushed and ground to a fine powder prior to measurement. The diffractometer was operated in high resolution capillary mode and with $2 \theta$ range of 0.001 to $149^{\circ}$. Variable temperature measurements were collected on cooling between $500-100 \mathrm{~K}$ with sample temperature controlled by a Cryostream Plus. Data were collected for either 30 minute durations for structural characterization or 15 minutes to enable fast collection of data for lattice parameter trends. Powder neutron diffraction was performed on the GEM instrument at the ISIS neutron source (Oxfordshire, UK). ${ }^{42}$ The powdered samples were loaded in vanadium cans which were mounted in a cryostat or furnace for variable temperature measurements, with data collected while heating between $50-600 \mathrm{~K}$.

For electrical characterization, the circular faces of sintered pellets were polished using silicon carbide abrasive paper and platinum or gold electrodes applied using an Emitech K550X sputter coater. Dielectric measurements were performed between 50 and $800 \mathrm{~K}$ with Agilent 4294A and Wayne Kerr 6500B impedance analyzers over the frequency range 0.025 - $2000 \mathrm{kHz}$. Samples were subjected to heating/cooling rates of $2 \mathrm{~K} \mathrm{~min}^{-1}$ in a closed cycle cyrocooler or tube furnace. Polarization-electric field $(P-E)$ data were measured with an aixACCT TF2000 Analyzer while the sample was mounted in an aixACCT piezo sample holder (model TFA 317-7) or closed cycle cryocooler.

Electron microscopy investigation was performed on a JEOL-2011 electron microscope operating at an accelerating voltage of $200 \mathrm{kV}$. Selected area electron diffraction (SAED) patterns and high-resolution transmission electron microscopy (HRTEM) images were recorded with a Gatan 794 CCD camera. 


\section{Results}

\section{A. $\mathrm{Ba}_{4} \mathrm{La}_{0.67} \mathrm{Nb}_{10} \mathrm{O}_{30}(\mathrm{x}=0)$}

The dielectric data for $\mathrm{Ba}_{4} \mathrm{La}_{0.67} \mathrm{Nb}_{10} \mathrm{O}_{30}(x=0)$ display both a low temperature relaxor-like response at low temperature $\left(\mathrm{T}_{\mathrm{m}}\right.$, at $297 \mathrm{~K}$ for $\left.1 \mathrm{MHz}\right)$ and a second diffuse but frequency independent peak in the relative permittivity at $471 \mathrm{~K}(1 \mathrm{MHz})$, Figure 2(a). A small amount of thermal hysteresis is observed (typically 10-15 K) in the low temperature anomaly; none is observed in the high temperature peak. Both the absolute, $\varepsilon$ ", and relative dielectric losses, $\tan \delta$, are dominated by a peak at low temperature corresponding to the relaxor-peak observed in the permittivity. The frequency and temperature dependence of the relaxor peak at low temperature is significantly greater than typically observed in both archetypal perovskite relaxors $^{2,7}$ and other TTBs with relaxor properties. ${ }^{43,44}$

The low temperature dielectric loss peak decreases in magnitude with increasing frequency which is contrary to the properties of relaxors with perovskite structure, as well as other reported TTBs. As a result of the coinciding decrease in permittivity with increasing frequency, a maximum in $\tan \delta$ occurs at approximately $240 \mathrm{~K}$. The high temperature permittivity peak displays no frequency dependence while the corresponding dielectric loss peak is small in magnitude by comparison, Figure 2(a) (inset). It should be noted that the dramatic increase in the low frequency permittivity observed at the highest temperatures is a result of the encroaching grain boundary/electrode polarization response. ${ }^{45}$ High temperature immittance spectroscopy shows that fixed frequency measurements at $1 \mathrm{MHz}$ remain on the plateau representing the bulk contribution to high temperature, $c a .850 \mathrm{~K}^{40}$

In an attempt to identify if either of the two maxima in the dielectric data were associated with a structural transformation, variable temperature powder neutron diffraction (PND) and synchrotron powder x-ray diffraction (s-PXRD) data were collected for $\mathrm{Ba}_{4} \mathrm{La}_{0.67} \mathrm{Nb}_{10} \mathrm{O}_{30}$. Initial inspection of both data sets revealed the deficiency of the previous assignment of tetragonal symmetry based on (lower resolution) ambient temperature laboratory PXRD data; ${ }^{40}$ the structure is orthorhombic over the entire measured temperature range between 20 $-600 \mathrm{~K}$. Closer inspection of the PND data revealed additional peaks of low intensity at longer d-spacing which do not correspond to likely impurity phases but which indicate the presence of a large orthorhombic superstructure. This superstructure is consistent with the Bbm2 model of Labbé and co-workers ${ }^{38}$ which has $a \approx 2 \sqrt{2} a_{T T B}, b \approx \sqrt{2} a_{T T B}$ and $c \approx 2 c_{T T B}$ expansions, Figure 3(a), and is the same structure reported for $\mathrm{Ba}_{4} \mathrm{La}_{2} \mathrm{Ti}_{4} \mathrm{Nb}_{6} \mathrm{O}_{30}{ }^{21}$ Rietveld refinement of the low temperature data PND data indicates that the atom positions in this compound are close to those determined for $\mathrm{BNN}$, see electronic supporting information (ESI) Figures S1-2 and Tables S1-3. It is important to note that due to the size of the structure and presence of 55 crystallographically distinct atoms, although it is possible to refine atom positions and individual isotropic displacement parameters, care should be taken not to place too much emphasis on the latter as these two variables are highly correlated. Note that refinement of PND data also supports sole occupancy of the A2 and A1 sites by Ba and the rare earth, consistent with our earlier study. ${ }^{40}$ 
The Bbm2 structure is generated primarily via the anti-phase tilts of the oxygen octahedra giving rise to two separate perovskite-site tilting patterns. The octahedral tilting is primarily out of the $a b$ plane. As the superstructure reflections are barely perceptible above the background of the synchrotron data in comparison to the PND data this suggests that the oxygen positions are primarily responsible for the superstructure. This is because of the insensitivity to oxygen positions of X-ray (even synchrotron X-ray) scattering compared with neutron scattering.

However, the fast data collection and high resolution of s-PXRD allows highly accurate cell parameter trends to be obtained. The data were refined in Bbm2 as determined by combined examination of s-PXRD and PND data (ESI Figures S1, S4-5). All cell dimensions exhibit linear thermal expansion with increasing temperature; however, two clear regimes are apparent with differing rates of expansion, Figure 3(c) and (d) and ESI Table S4. The different rates of thermal expansion in $a$ and $b$, reduces the orthorhombicity of the structure with increasing temperature, Figure 3(d). The change in expansion rate and orthorhombicity coincides with the low temperature relaxor-like peak $(250-270 \mathrm{~K})$, however, the diffraction data provides no evidence of a symmetry change at these temperatures. A symmetry transformation may be present but imperceptible due to the structural complexity; several perovskite relaxors appear to be cubic at temperatures above and below the respective relaxor peak due to spatial averaging associated with bulk diffraction techniques. ${ }^{2,8,46,47}$

The intensity of the superstructure peaks in the neutron data decreases with increasing temperature and are undetectable by $480 \mathrm{~K}$ indicating disappearance of the superstructure, Figure 3 (b). The high temperature, frequency independent peak in the dielectric data corresponds with this loss of superstructure indicating a transition from the low temperature $B b m 2$ superstructure to a simpler high temperature orthorhombic structure with comparable metrics to the aristotype cell (i.e. no superstructure is detectable using PND). Refinements using the simple orthorhombic Pbam structure, previously reported for $\mathrm{Pb}_{4.5} \mathrm{Ca}_{0.5} \mathrm{Ta}_{10} \mathrm{O}_{30}{ }_{48}$ and $\mathrm{KFe}_{2} \mathrm{~F}_{6}{ }^{49}$, result in good agreement between calculated and observed patterns (ESI Figure S3), however, further study of the structure with e.g. high temperature SAED etc. would be required to conclusively assign this space group. It should be noted that based on P-E data (discussed later) the material is not polar above $480 \mathrm{~K}$ - this is consistent with the assignment of Pbam symmetry.

Selected area electron diffraction (SAED) of the $\mathrm{x}=0$ sample taken down the $[\overline{1} 12]_{\text {ттв }}$ zone axis is shown in Figure 4 (a). The presence of additional satellite diffraction spots confirms the presence of a supercell and indexing is consistent with the assignment of the space group based on the diffraction data discussed above. The zone axis according to the $2 \sqrt{ } 2 a_{T T B} \times$ $\sqrt{2} b_{T T B} \times 2 c_{T T B}$ supercell is therefore $[0 \overline{1} 1]_{B b m 2}$. The even spacing of the satellite peaks indicates that the structure is commensurate. For example, the marked spots A and B can be indexed to (111) and (311), respectively. SAED pattern taken down the $[\overline{1} 11]_{B b m 2}$ zone axis (Figure $4 \mathrm{~b}$ ) shows diffused diffraction spots along the [110] direction. Diffuse spots along [250] direction (Figure 4c) are also observed. This indicates disorder in the $a b$ plane and as a result no additional superstructure can be derived; there is no evidence of any disorder in the $c$-axis. As a result the structure of $\mathrm{Ba}_{4} \mathrm{La}_{0.67} \mathrm{Nb}_{10} \mathrm{O}_{30}$ is described as commensurate $\mathrm{Bbm} 2$ but 
with disorder. This in contrast to the filled TTB $\mathrm{Ba}_{4} \mathrm{La}_{2} \mathrm{Ti}_{4} \mathrm{Nb}_{6} \mathrm{O}_{30}$ which adopts the same structure, but which has a modulation of the oxygen positions as a result of frustrated tilts and is incommensurate. ${ }^{21}$ The presence of an incommensurate structure was attributed to be the origin of relaxor behavior in this compositional series, while commensurate (ordered) compositions were normal ferroelectrics. In order to further investigate the influence of the slightly differing crystallography on the electrical behavior of these closely-related TTBs the thermal dependence of the ferroelectric hysteresis was investigated.

Polarization-field (P-E) data and the corresponding current (I-E) responses are shown as a function of temperature for $\mathrm{Ba}_{4} \mathrm{La}_{0.67} \mathrm{Nb}_{10} \mathrm{O}_{30}$ in Figure 5. At low temperature, below the onset of the relaxor-type peak in the permittivity, normal, well-saturated ferroelectric hysteresis loops are observed. On heating the loops become increasingly slim and develop a pinched or constricted appearance between $c a .260$ and $470 \mathrm{~K}$ and indicate the loss of ferroelectric behavior. Above $470 \mathrm{~K}$, the P-E (and I-E) data indicate a linear, slightly lossy, dielectric response consistent with a non-polar phase. The nature of the development of this "pinched" non-ferroelectric behavior is evident in the I-E data. An additional "backswitching" event occurs on unloading as indicated by the current peaks marked in Figure 5; the field against which the sample "back-switches" or "depolarizes" is denoted $+E_{D}$. This depolarization event emerges at low fields initially then to higher fields with increasing temperature. P-E loops and I-E data were collected at varying electric fields up to values sufficient large enough to cause dielectric breakdown; the position of the maxima on unloading in the I-E data and the pinched morphology of the P-E loops is independent of the magnitude and frequency of the applied field.

The ferroelectric P-E loop indicates polar ordering at low temperature consistent with a relaxor ferroelectric (RFE), rather than a relaxor dielectric, dipole glass-like, response. The transformation to the pinched loop is correlated with changes in both structural and dielectric data. While no distinct phase change is detected by diffraction this may be due to a change from a locally ordered polar to 'non-polar' structure due to disorder as indicated by in SAED. In many perovskite-based relaxors no long-range symmetry breaking is observed despite the formation of local regions of non-centrosymmetry (i.e., polar nano-regions, PNRs) but they do not display similar dielectric behavior to that observed here. The disordered phase in $\mathrm{Ba}_{4} \mathrm{La}_{0.67} \mathrm{Nb}_{10} \mathrm{O}_{30}$ appears to retain average polar symmetry and develop a large polarization (but not necessarily ferroelectric behavior) with the application of an external electric field note the saturated polarization is similar in magnitude in both the low temperature RFE regime and disorder "pinched-loop" regime. The subsequent loss of superstructure reflections, peak in permittivity, together with linear dielectric P-E measurements at $473 \mathrm{~K}$, indicate a subsequent phase transition and place an upper temperature limit for this region of unusual behavior.

The temperature dependence of the electric field responsible for ferroelectric switching (coercive field, $\mathrm{E}_{\mathrm{C}}$ ) and the backswitching (depolarization, $\mathrm{E}_{\mathrm{D}}$ ) events is shown in Figure 6(a). The coercive field, $E_{C}$, for the normal FE P-E loops follows the usual temperature dependence and decreases with increasing temperature. At the onset of the region where pinched loops are observed, both the coercive $\left(E_{C}\right)$ and depolarization $\left(E_{D}\right)$ fields increase 
with increasing temperature, demonstrating the increasing stability of the disordered regime. The minimum in the coercive field, the change in lattice parameter trends and a decrease in the remnant polarization, Figure 6(b), all coincide with the low temperature relaxor peak. The onset of pinching may therefore be attributed to the disordering process between the low temperature relaxor and high temperature permittivity peaks. At higher temperatures, the I-E response becomes increasingly broad making reliable determination of $\mathrm{E}_{\mathrm{C}}$ and $\mathrm{E}_{\mathrm{D}}$ difficult.

Such pinched P-E loops have been previously reported in perovskites and have been variously attributed to: aging, antiferroelectric ordering, defect-dipole pairs, and field driven ferroelectric phase transitions. ${ }^{50-54}$ Thermal annealing above $\mathrm{T}_{\mathrm{C}}$ or applying 'de-aging' or fatiguing bipolar excitation cycles of sufficient field can reverse the mechanisms responsible for aged, defect-mediated pinched P-E loops in "normal" ferroelectrics. ${ }^{55-57}$ Such measurements were performed on $\mathrm{Ba}_{4} \mathrm{La}_{0.67} \mathrm{Nb}_{10} \mathrm{O}_{30}$ (see ESI Figures $\mathrm{S} 12-\mathrm{S} 15$ for details). Little effect on the P-E response was identified and the pinched character remained, therefore aging/defect-dipole pairs is unlikely to be the source of the observed P-E behavior here. In addition, SAED provided no evidence for phase co-existence nor evidence of PNRs. If the pinched loop behavior results from antiferroelectric ordering or growth of PNRs, these must exist on very short lengths scales.

\section{B. $\mathrm{Ba}_{4} \mathrm{Nd}_{0.67} \mathrm{Nb}_{10} \mathrm{O}_{30}(\mathrm{x}=1)$}

Dielectric data indicate a single peak in the relative permittivity for $\mathrm{R}=\mathrm{Nd}$ which exhibits only a small degree of frequency dependence (peak maximum measured between $399 \mathrm{~K}$ at 1 $\mathrm{kHz}$ and $405 \mathrm{~K}$ at $1 \mathrm{MHz}$ c.f. $x=0,242 \mathrm{~K}$ and $297 \mathrm{~K}$, respectively), Figure 2 (b). No thermal hysteresis is observed between heating and cooling measurements. The dielectric loss is dominated by maxima corresponding to $T_{C}$ with an additional 'shoulder' at lower temperature. P-E data indicate $\mathrm{Nd}$ to be ferroelectric at all measured temperatures up to $T_{C}$ then (slightly lossy) linear dielectric behavior at high temperature - no constriction of the loops was observed (see ESI Figure S8). This represents a normal ferroelectric to paraelectric transition although the loops are slimmer than other compositions in this empty series. ${ }^{40}$

Ambient temperature synchrotron data for $x=1$ confirms the larger 'tetragonality', $c / a$ as previously reported based on a reduced unit cell comparable with the TTB aristotype. When indexed to an orthorhombic cell, $a$ and $b$ are both smaller for $x=1$ than comparable values for $x=0$. In addition, the orthorhombicity of the cell at ambient temperature is smaller orthorhombic strain of 0.064 compared to 0.081 for $x=1$. No evidence of a supercell was detected in the sPXRD data.

Corresponding ambient temperature PND data shows additional peaks at higher d-spacing, which are in similar positions to those observed for $x=0$, but of dissimilar intensities. Levin and co-workers report that $\mathrm{Ba}_{4} \mathrm{Nd}_{2} \mathrm{Ti}_{4} \mathrm{Nb}_{6} \mathrm{O}_{30}$ has the commensurate orthorhombic Ima 2 structure with $a \approx \sqrt{ } 2 a_{T T B}, b \approx \sqrt{2} b_{T T B}$ and $c \approx 2 c_{T T B}$, however, the required reflections were absent in our observed data. Reflections at these peak positions did appear to be satisfied by the Bbm2 model, however, the intensities and shapes of the peaks were poorly fitted. As with 
$x=0$, we were unable to reliably refine atom positions or individual isotropic displacement parameters which both contribute to intensity and peak shape due to the larger number of atoms in the unit cell and corresponding large number of refinement variables. Additionally, the oxygen modes coupled with the octahedral tilt, modulate the intensity of these superstructure reflections. This may suggest the structure is $B b m 2$ with the same pattern but slightly different extents of tilting. The larger Im $2 a\left(2 \sqrt{2} a_{T T B} \times 2 \sqrt{2} b_{T T B} \times 2 c_{T T B}\right)$ structure reported by Torres-Pardo and co-workers ${ }^{19}$ for commensurate $\mathrm{Sr}_{4} \mathrm{Na}_{2} \mathrm{Nb}_{10} \mathrm{O}_{30}$ (SNN) also generates a similar diffraction pattern. Due to the small number of reflections of sufficient intensity it is difficult to conclusively distinguish between these two cells from either sPXRD or PND data.

SAED patterns from the $x=1$ sample are similar to those from the $x=0$ sample. Figure 7 shows a typical HRTEM image and the corresponding SAED pattern viewing down the same

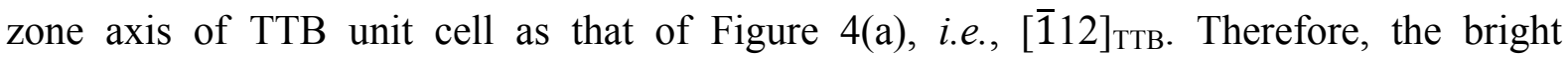
diffraction spots derived from the TTB basic unit cell form the same pattern. The satellite diffraction spots also have a similar pattern as that in Figure 4(a), however, in contrast to the measured inter-spot distances in Figure 4(a) those in Figure 7 are not uniform indicating that the compound is incommensurate. ${ }^{58,59}$ The incommensurability parameter, $\delta$, given by (b a) $/(b+a)=0.177$ is more similar in magnitude to $\delta \approx 0.19-0.20$ reported by Bursill and Lin for $\mathrm{SBN}^{24}$ than $\delta=0.04$ reported by Stennett et al. for $\mathrm{Ba}_{4} \mathrm{La}_{2} \mathrm{Ti}_{4} \mathrm{Nb}_{6} \mathrm{O}_{30}{ }^{31}$ Normally, the metal atoms make significant contribution to the SAED patterns of the basic TTB lattice, while the oxygen arrangement is responsible for the formation of the superstructure. Local changes of the oxygen environment may form some special clusters, which will give relatively strong diffraction contrast, forming some dark spots in the HRTEM images. We can also see atomic dislocations in these dark spots (left inset in Figure 7), a similar phenomenon has been observed as excess oxygen in perovskite structure, resulting in local distortion. ${ }^{60,61}$ Partial ordering of these clusters may form incommensurate structures. Nevertheless, this contrast pattern was not observed from HRTEM images from the $x=0$ sample. The presence of an incommensurate modulation may account for the weak frequency dependence observed in the permittivity temperature data consistent with the models of Levin et $a .^{21}$ and Zhu et al. ${ }^{20}$

The Nd-analogue $(x=1)$, therefore represents the transitional composition between RFE and normal ferroelectric behavior in this TTB series. The low field $\varepsilon^{*}(\mathrm{~T})$ data, particularly the loss, shows some differences to the other normal ferroelectric TTBs with A-cations smaller than Nd within this series. ${ }^{40}$ The normal P-E response observed in this composition may be due to stabilization of the ferroelectric phase during measurement.

\section{Intermediate compositions, $\mathrm{Ba}_{4}\left(\mathrm{La}_{1-\mathrm{x}} \mathrm{Nd}_{\mathrm{x}}\right)_{0.67} \mathrm{Nb}_{10} \mathrm{O}_{30}, \mathrm{x}=\mathbf{0 . 2 5}, \mathbf{0 . 5}, 0.75$}

In order to investigate further the transition from RFE to ferroelectric behavior with cation size, intermediate compositions with partial substitution of $\mathrm{La}$ and $\mathrm{Nd}$ were studied. From laboratory PXRD data of $x=0.25,0.5$ and 0.75 these compounds have cell dimensions and 'tetragonal' splitting which are intermediary of the two end members, although as previously 
reported the difference in such metrics is incredibly small between $\mathrm{La}$ and $\mathrm{Nd} .{ }^{40}$ No evidence was found for the presence of secondary phases or phase separation, which has previously been identified in a number of different TTBs. ${ }^{62,63}$ Ambient temperature PND data of $x=0.5$ evidences the existence of a superstructure similar to the end members. As with $x=1$, it is not possible to distinguish between Bbm2 and the larger Im $2 a$ cell. Qualitatively, it appears to be an intermediate between the $x=0$ and 1 patterns.

The permittivity data of the intermediate compounds shows decreasing relaxation and diffuseness of the low temperature anomaly with increasing $x$, while the maximum simultaneously shifts to higher temperature, Figure 8 (and ESI Figures S6 and S7). The high temperature peak decreases in both magnitude and temperature, until both peaks coalesce for $x=1$. At lower $x$ values, the dielectric loss is dominated by the low temperature response corresponding to the relaxor-like behavior in the permittivity.

P-E measurements performed on these compounds, (ESI Figures S8-S11), demonstrate that all compounds are ferroelectric below the relaxor-like peak or peak maximum $\left(T_{C}\right)$ for higher $x$. This corresponds with the approximate temperatures of the largest maxima in the dielectric loss. For lower $x$ values $(x=0.25$ and 0.5$)$ pinched P-E loops were recorded at temperatures between the two peaks in the relative permittivity i.e., at temperatures above the RFE phase and below the high temperature non-polar phase. The coalescence of these peaks with increasing $x$, leads to a decrease in temperature ranges over which the disordered phase is stable; for $x=0.25$ it persists over a wide temperature range $(>100 \mathrm{~K})$ while for $x=0.5$ pinched loops are recorded over only a very narrow range. The pinched character of the P-E loop also decreases with increasing $x$ - the effect is most evident for $x=0$. Pinched P-E loop behavior is absent for higher $x$ values; for $x=0.75$ there is a direct transition from ferroelectric P-E loop to standard linear dielectric type indicating a ferroelectric to non-polar paraelectric transition as described for $\mathrm{Ba}_{4} \mathrm{Nd}_{0.67} \mathrm{Nb}_{10} \mathrm{O}_{30}(x=1)$, above.

\section{Discussion}

The presence of both frequency dependent, relaxor-type and normal, frequency independent peaks have been observed in the permittivity-temperature profile of a number of TTB compositions. ${ }^{19,}$ 37, ${ }^{64-71}$ However, the relaxor-like response in these is often subtle or convoluted with the main permittivity peak associated with $T_{C}$ and has not been demonstratively linked changes in the structural or P-E properties and so the origins of such behavior has not been convincingly assigned. The pronounced separation of the features presented here for $\mathrm{Ba}_{4} \mathrm{La}_{0.67} \square_{1.33} \mathrm{Nb}_{10} \mathrm{O}_{30}$ in conjunction with observed structural variations and changes in P-E behavior provides a valuable opportunity to elucidate the origins of such behavior in the context of the recent crystal-chemical model for relaxor versus normal ferroelectric behavior as a function of composition in TTBs. ${ }^{20}$ In that model, the observed behavior is determined by a subtle competition between structural modulations, specifically octahedral tilting as determined by oxygen positions, and polarization generated by B-cation displacements. To summarise, small A-cation sizes, and hence low tolerance factor of the perovskite unit, results in strong octahedral tilting in a coherent, commensurate manner and 
so long range order and normal ferroelectric behavior is observed. With increasing A-cation size the degree of tilting decreases and the tilt system becomes increasingly frustrated, denoted by an increasing distribution of the oxygen positions, and reduction in symmetry. The variation in oxygen positions retains a degree of periodicity and so an incommensurate structure and relaxor behavior is observed. However, for particularly large A-cations the extension of the $\mathrm{O}-\mathrm{B}-\mathrm{O}$ bonds stabilize the polarization and overcome the disruption of long range order due to the incommensurate structure and normal ferroelectric behavior is again observed.

The results presented here are broadly consistent with this model and it is possible to rationalize the observed dielectric and structural behavior in that context. According to our model, and based on the chemical composition of $\mathrm{Ba}_{4} \mathrm{La}_{0.67} \square_{1.33} \mathrm{Nb}_{10} \mathrm{O}_{30}$ one would predict an incommensurate structure and relaxor behavior as exhibited by the filled analogue $\mathrm{Ba}_{4} \mathrm{La}_{2} \mathrm{Ti}_{4} \mathrm{Nb}_{6} \mathrm{O}_{30}$, and that by decreasing the $\mathrm{A} 1$ cation size one can induce normal ferroelectric behaviour. $\mathrm{Ba}_{4} \mathrm{La}_{0.67} \square_{1.33} \mathrm{Nb}_{10} \mathrm{O}_{30}$ is indeed a relaxor, but it appears however, that the presence of a significant degree of A-site vacancies allows enough structural relaxation that the disorder results rather than an incommensurate modulation. For the Ndanalogue the decreased A-cation size promotes a larger degree of tilting and re-introduces a degree of periodic modulation and this composition is an incommensurate ferroelectric. The compositions $\mathrm{Ba}_{4} \mathrm{La}_{0.67} \square_{1.33} \mathrm{Nb}_{10} \mathrm{O}_{30}$ and $\mathrm{Ba}_{4} \mathrm{Nd}_{0.67} \square_{1.33} \mathrm{Nb}_{10} \mathrm{O}_{30}$ therefore represent a crossover between commensurate, relaxor ferroelectric and incommensurate, ferroelectric phases and further highlight the key role of $a b$ tilt modulations in determining the properties of these, and related (e.g. filled), TTB compositions. The role of the A1 vacancies, therefore, appears to be to add an additional disruption to the lattice which perturbs the structural variations to a greater extent than in the filled analogous compositions.

For $\mathrm{Ba}_{4} \mathrm{La}_{0.67} \square_{1.33} \mathrm{Nb}_{10} \mathrm{O}_{30}$, the SAED data provide evidence for disorder which, in turn, disrupts long range polar ordering. However, the temperature dependence of both the relative permittivity and P-E behavior suggest that this disorder is rather weak. At low temperature, normal P-E behavior and the presence of a persistent remanent polarization confirms long range, net polarization and suggest that the oxygen positions order (incommensurately) at low temperature, resulting in a modulated tilt system. On heating, the large peak in the dielectric loss combined with a loss of remanent polarization, as demonstrated by the pinched P-E hysteresis behavior, is consistent with the onset of disorder observed in the SAED. This also results in a subtle change in the thermal expansion in long range, average structure as determine by other diffraction methods. In this intermediate temperature regime, the high polarizability of the oxygen sublattice allows application of a sufficiently large electric field to induce long range order, as indicated by the switch event in the P-E data on loading and the similarity in the maximum induced polarization $\left(P_{\max } \sim 6 \mu \mathrm{C} . \mathrm{cm}^{-2}\right)$ at maximum field $(E \sim$ $\left.100 \mathrm{kV} . \mathrm{cm}^{-1}\right)$, Figure 5, but which relaxes on removal of the field. The data in Figure $6(\mathrm{a})$ suggests that the stability of disordered regime increases with increasing temperature, with both the coercive field required for ordering $\left( \pm E_{C}\right)$ and field against which relaxation occurs $\left( \pm E_{D}\right)$ increases with increasing temperature, until the paraelectric phase transition is reached at $c a .500 \mathrm{~K}$. 
Double or pinched loops have also been reported in a number of perovskite systems with a number of mechanisms suggested. Antiferroelectrics (AFEs) produce double loops due to an electric field-induced transition between AFE and ferroelectric phases of similar energy. ${ }^{52}$ Such loops, first demonstrated in $\mathrm{PbZrO}_{3}$ by Shirane et al. ${ }^{50,51}$ are characteristic of AFE ordering and consequently many systems were designated as being AFE based on P-E loops. Few instances of pinched "antiferroelectric" loops have been reported in TTBs. ${ }^{72-75}$ An AFEFE-PE phase transition sequence was described by Ravez and colleagues in the TTB-like compounds in the solid solution $\mathrm{Ba}_{2.14} \mathrm{Li}_{0.71} \mathrm{Nb}_{5} \mathrm{O}_{15}-\mathrm{Ba}_{2.14} \mathrm{Li}_{0.71} \mathrm{Ta}_{5} \mathrm{O}_{15}$ (i.e. $\left.\mathrm{Ba}_{4.28} \mathrm{Li}_{1.42} \mathrm{Nb}_{10} \mathrm{O}_{30}-\mathrm{Ba}_{4.28} \mathrm{Li}_{1.42} \mathrm{Ta}_{10} \mathrm{O}_{30}\right) .{ }^{17,}{ }^{72} \mathrm{Gagou}$ et al. have recently attributed two dielectric anomalies in $\mathrm{K}_{4} \mathrm{Gd}_{2} \mathrm{Nb}_{10} \mathrm{O}_{30}$ to a FE-AFE-PE sequence of phase transitions based on Raman spectroscopy data, ${ }^{74}$ however, no P-E loops were demonstrated and the low temperature anomaly has previously been associated with a ferroelastic related transition between orthorhombic (pseudo-tetragonal) and tetragonal structures. ${ }^{18}$ Pinched P-E loops have been reported in $\mathrm{La}$ and $\mathrm{Ti}$ co-doped $\mathrm{SBN}, \mathrm{Sr}_{0.25} \mathrm{Ba}_{0.7} \mathrm{La}_{0.03} \mathrm{Nb}_{2-\mathrm{y}} \mathrm{Ti}_{\mathrm{y}} \mathrm{O}_{6-\delta}$ with $\mathrm{y}=0.05$ (i.e. $\mathrm{Sr}_{1.275} \mathrm{Ba}_{3.5} \mathrm{La}_{0.15} \mathrm{Nb}_{9.75} \mathrm{Ti}_{0.25} \mathrm{O}_{30-\delta}$ ) in which $\mathrm{FE}$ and AFE states coexist. The authors suggest a polar FE matrix with commensurate tetragonal structure within which local ordering of an incommensurately modulated AFE structure occurs. ${ }^{73} \mathrm{Ti}^{4+}$ dopant-induced defects were suggested as being responsible for the weakening of the polar FE ordering, favoring the formation of the AFE state. ${ }^{73}$ However, the required crystallographic anti-polar arrangement for antiferroelectricity is not evident in the compositions studied here and so long range AFE order can be discounted.

Other mechanisms shown to affect such P-E behavior include: double loops within a narrow temperature range close to $\mathrm{T}_{\mathrm{C}}$ due to an electric field driven transition between the paraelectric and ferroelectric phases in ferroelectrics with a sharp first order ferroelectricparaelectric phase transition (e.g. undoped $\left.\mathrm{BaTiO}_{3}\right) ;{ }^{54}$ defect dipoles in aged ferroelectrics. ${ }^{53}$, ${ }^{76}$ Based on the observations reported here and presented in the ESI, we also discount these mechanisms.

$\mathrm{Bi}_{0.5} \mathrm{Na}_{0.5} \mathrm{TiO}_{3}$ (BNT) and solid solutions $\left(\mathrm{BaTiO}_{3}\right.$-BNT and $\left.\mathrm{Bi}_{0.5} \mathrm{~K}_{0.5} \mathrm{TiO}_{3}-\mathrm{BNT}\right)$ show similar temperature dependent dielectric properties. with a frequency dependent relaxor-like peak around $473 \mathrm{~K}$ in undoped BNT and high temperature peak with little dispersion $(\approx 593$ $\mathrm{K}){ }^{77-79}$ BNT compounds have a complex sequence of phase transitions and may include coexisting phases. ${ }^{77-80}$ The presence of an AFE phase was proposed due to pinched/doubled P-E loops, however other explanations have since been offered involving coexisting phases, domains or nano-regions of different symmetries and electric field driven transitions between a weakly or non-polar relaxor phase and that of a polar ferroelectric phase. ${ }^{77-84}$ The origin for these properties is still somewhat contested and may differ in each respective solid solution. ${ }^{83}$ From SAED we see no evidence of PNRs or subtle compositional segregation in our materials.

Therefore, based on the observations in the compositions presented here, the frequency dependent, relaxor-type and frequency independent, peaks in permittivity observed on heating indicate transitions from incommensurate, relaxor ferroelectric $(B b m 2)$ to disordered, non-ferroelectric (Bbm2) to paraelectric (Pbam) phases. The $\mathrm{Ba}_{4}\left(\mathrm{La}_{1-\mathrm{x}} \mathrm{Nd}_{\mathrm{x}}\right)_{0.67} \square_{1.33} \mathrm{Nb}_{10} \mathrm{O}_{30}$ 
compositional series show a decrease in temperature range between these transitions being "closed out', indicating a destabilization of the disordered phase until normal incommensurate ferroelectric behavior with no pinching is observed in compositions with $x>$ 0.5 . It is important to re-emphasize that for $x=0.25$ and $x=0.5$, pinched P-E behavior is observed in the temperature range between permittivity peaks; the fact that this is not observed for $x=0.75$ despite evidence of a double permittivity peak suggests that the disorder is easily overcome by even modest applied fields.

The data indicate the role of vacancies in allowing a structural relaxation to de-stabilize long range polar ordering, but only to the extent that it can be re-introduced by a sufficiently large electric field. The resulting behavior is a pinched P-E response with the same $P_{\max }$ as the FE phase but with no significant remnant polarization. Such behavior is one of the key signatures currently being sought for exploitation of "ferroelectric" materials for energy harvesting capacitors. Although similar pinched loop behavior has also been observed in simple perovskites such as BNT, the origins of the observed behavior in that system is still under debate. Here we show that A-site vacancies in the perovskite unit of the TTB structure provides a tool to develop materials on the edge of ferroelectric stability and which could allow further exploitation of these materials for e.g. energy harvesting.

\section{Conclusions}

The "empty" tetragonal tungsten bronze (TTB) $\mathrm{Ba}_{4} \mathrm{La}_{0.67} \mathrm{Nb}_{10} \mathrm{O}_{30}$ exhibits both frequency dependent, relaxor-type ( $c a .260 \mathrm{~K})$ and frequency independent $(c a .480 \mathrm{~K})$ peaks in the dielectric data. While similar behavior has been previously observed in other TTBs the behavior is not as evident, nor has this relaxor feature been clearly associated with any structural variation or change in the polarization-field (P-E) behavior. Here we clearly demonstrate that heating through the relaxor-like peak is associated both with the loss of ferroelectric switching and onset of a pinching of the P-E response and also a change in the crystal structure as demonstrated by both synchrotron $\mathrm{x}$-ray and neutron powder diffraction. In addition, diffuse scattering in selected area electron diffraction indicates that in the region where pinched P-E loops are observed, disorder exists. This structural disorder disrupts long range polar ordering and accounts for the observed loss of ferroelectricity. Application of an electric field reintroduces a large non-linear polarization of the same magnitude as in the ferroelectric phase, but on removal of the field, non-linear depolarization signifies a "backswitching" event associated with the structural relaxation to the disordered phase. This backswitching event occurs against and increasingly large electric field with increasing temperature indicating increased stability of the disorder with increasing thermal energy. The presence of a large concentration of vacancies at the A1-site facilitates the structural relaxation and disorder and is the reason why the effects are more evident in this compositional series.

The temperature range over which the disordered phase and pinched P-E persists can be reduced by replacement of La with smaller $\mathrm{Nd}$ cations at the A1-site (increasing $x$ in $\mathrm{Ba}_{4}\left(\mathrm{La}_{1}\right.$ $\left.{ }_{x} \mathrm{Nd}_{\mathrm{x}}\right)_{0.67} \square_{1.33} \mathrm{Nb}_{10} \mathrm{O}_{30}$ ). With increasing $x$ the tolerance factor of the perovskite unit decreases 
and induces an increase in tilting of the $\mathrm{NbO}_{6}$ octahedra. As a result, the temperature range over which pinched P-E behavior is observed decreases until for $x=1$ $\left(\mathrm{Ba}_{4} \mathrm{Nd}_{0.67} \square_{1.33} \mathrm{Nb}_{10} \mathrm{O}_{30}\right)$ a weakly frequency dependent, relaxor-like permittivity peak and normal ferroelectric switching is observed.

The influence of high concentrations $(>50 \%)$ of A1-site vacancies in these materials provides further insights into our understanding of structure-property relationships in TTB materials.

\section{Acknowledgements}

We thank both ISIS neutron and muon facility for the use of the GEM instrument (RB1310284) and Diamond Light Source for access to beamline I11 (EE9331) that contributed to the results presented here. JG would like to thank the EPSRC for provision of a studentship via the doctoral training grant (EP/K503162/1). The research data (and/or materials) supporting this publication can be accessed at [http://dx.doi.org/10.17630/a7a9bc13-b5cb-485d-914a-923832f25190]

\section{Supplementary Information}

Structural data including Rietveld refinements of PND and SPXRD data; linear thermal expansion coefficients; dielectric data; polarization-field measurements including after ageing and quenching of samples.

(1) Bhalla, A. S.; Guo, R. Y.; Roy, R., The perovskite structure - a review of its role in ceramic science and technology. Mater. Res. Innovations 2000, 4, 3-26.

(2) Bokov, A. A.; Ye, Z. G., Recent progress in relaxor ferroelectrics with perovskite structure. J. Mater. Sci. 2006, 41, 31-52.

(3) Haertling, G. H., PLZT electrooptic materials and applications-a review. Ferroelectrics 1987, 75, 25-55.

(4) Davies, P. K.; Akbas, M. A., Chemical order in PMN-related relaxors: structure, stability, modification, and impact on properties. J. Phys. Chem. Solids 2000, 61, 159-166.

(5) Stenger, C. G. F.; Burggraaf, A. J., Order-disorder reactions in the ferroelectric perovskites $\mathrm{Pb}(\mathrm{Sc} 1 / 2 \mathrm{Nb} 1 / 2) \mathrm{O} 3$ and $\mathrm{Pb}(\mathrm{Sc} 1 / 2 \mathrm{Ta} 1 / 2) \mathrm{O} 3$. II. Relation between ordering and properties. Phys. Status Solidi A 1980, 61, 653-664.

(6) Setter, N.; Cross, L. E., The role of B-site cation disorder in diffuse phase transition behavior of perovskite ferroelectrics. J. Appl. Phys. 1980, 51, 4356.

(7) Bokov, A. A.; Ye, Z.-G., Dielectric Relaxation in Relaxor Ferroelectrics. J. Adv. Dielectr. 2012, 02, 1241010.

(8) Shvartsman, V. V.; Lupascu, D. C., Lead-Free Relaxor Ferroelectrics. J. Am. Ceram. Soc. 2012, 95, 1-26.

(9) Chen, I. W., Structural origin of relaxor ferroelectrics - Revisited. J. Phys. Chem. Solids 2000, 61, 197-208.

(10) Cross, L. E., Relaxor ferroelectrics: An overview. Ferroelectrics 1994, 151, 305-320. 
(11) Hirota, K.; Wakimoto, S.; Cox, D. E., Neutron and X-ray scattering studies of relaxors. J. Phys. Soc. Jpn. 2006, 75.

(12) Jamieson, P. B.; Abrahams, S. C.; Bernstein, J. L., Ferroelectric tungsten bronze-type crystal structures. II. Barium sodium niobate $\mathrm{Ba}(4+\mathrm{x}) \mathrm{Na}(2-2 \mathrm{x}) \mathrm{Nb} 10 \mathrm{O} 30$. J. Chem. Phys. 1969, 50, 4352-4363.

(13) Glass, A. M., Investigation of the Electrical Properties of Sr1-x Ba x NB2O6 with special reference to pyroelectric detection. J. Appl. Phys. 1969, 40, 4699-4713.

(14) Jamieson, P. B.; Abrahams, S. C.; Bernstein, J. L., Ferroelectric tungsten Bronze-type crystal structures. I. Barium strontium niobate Bao.27Sr0.75Nb2O5.78. J. Chem. Phys. 1968, 48, 5048-5057.

(15) Abrahams, S. C.; Jamieson, P. B.; Bernstein, J. L., Ferroelectric tungsten bronze-type crystal structures. III. Potassium lithium niobate K6-x-y $\} \operatorname{Li}(4+x) \mathrm{Nb}(10+y) \mathrm{O} 30$. J. Chem. Phys. 1971, 54, 2355-2364.

(16) Lines, M. E.; Glass, A. M., Principles and Applications of Ferroelectrics and Related Materials Oxford : Clarendon 2001.

(17) Simon, A.; Ravez, J., Solid-state chemistry and non-linear properties of tetragonal tungsten bronzes materials. C. R. Chim. 2006, 9, 1268-1276.

(18) Neurgaonkar, R. R.; Nelson, J. G.; Oliver, J. R.; Cross, L. E., Ferroelectric and structural properties of the tungsten bronze system $\mathrm{K} 2 \mathrm{Ln} 3+\mathrm{Nb} 5 \mathrm{O} 15, \mathrm{Ln}=\mathrm{La}$ to Lu. Mater. Res. Bull. 1990, 25, 959-970.

(19) Torres-Pardo, A.; Jimenez, R.; Gonzalez-Calbet, J. M.; Garcia-Gonzalez, E., Structural Effects Behind the Low Temperature Nonconventional Relaxor Behavior of the Sr2NaNb5O15 Bronze. Inorg. Chem. 2011, 50, 12091-12098.

(20) Zhu, X.; Fu, M.; Stennett, M. C.; Vilarinho, P. M.; Levin, I.; Randall, C. A.; Gardner, J.; Morrison, F. D.; Reaney, I. M., A Crystal-Chemical Framework for Relaxor versus Normal Ferroelectric Behavior in Tetragonal Tungsten Bronzes. Chem. Mater. 2015, 27, 3250-3261.

(21) Levin, I.; Stennett, M. C.; Miles, G. C.; Woodward, D. I.; West, A. R.; Reaney, I. M., Coupling between octahedral tilting and ferroelectric order in tetragonal tungsten bronzestructured dielectrics. Appl. Phys. Lett. 2006, 89.

(22) Graetsch, H. A.; Pandey, C. S.; Schreuer, J.; Burianek, M.; Mühlberg, M., Incommensurate modulations of relaxor ferroelectric $\mathrm{Ca} 0.24 \mathrm{Ba} 0.76 \mathrm{Nb} 2 \mathrm{O} 6(\mathrm{CBN} 24)$ and $\mathrm{Ca}$ 0.31Ba0.69Nb2O6 (CBN31). Acta Crystallogr., Sect. B: Struct. Sci., Cryst. Eng. Mater. 2014, 70, 743-749.

(23) Mao, M. M.; Li, K.; Zhu, X. L.; Chen, X. M., Incommensurate and commensurate modulations of Ba5RTi3Nb7O30 $(\mathrm{R}=\mathrm{La}, \mathrm{Nd})$ tungsten bronzes and the ferroelectric domain structures. J. Appl. Phys. 2015, 117, 134108.

(24) Bursill, L. A.; Lin, P. J., Incommensurate superstructures and phase transition of strontium barium niobate (SBN). Acta Crystallogr., Sect. B: Struct. Sci. 1987, 43, 49-56.

(25) Lin, P. J.; Bursill, L. A., Superlattice structure of ferroelectric barium sodium niobate (BNN). Acta Crystallogr., Sect. B: Struct. Sci. 1987, 43, 504-512.

(26) Schneck, J.; Toledano, J. C.; Joffrin, C.; Aubree, J.; Joukoff, B.; Gabelotaud, A., Neutron-Scattering Study of the Tetragonal-to-Incommensurate Ferroelastic Transition in Barium Sodium Niobate. Phys. Rev. B Condens. Matter Mater. Phys. 1982, 25, 1766-1785.

(27) Schneck, J.; Toledano, J. C.; Errandonea, G.; Litzler, A.; Savary, H.; Manolikas, C.; Kiat, J. M.; Calvarin, G., Coexistence of 2 Phases in Incommensurate Barium Sodium Niobate. Phase Transitions 1987, 9, 359-364.

(28) Kiat, J. M.; Calvarin, G.; Schneck, J., Coexistence of the 1q and 2q incommensurate phases and memory effect in barium sodium niobate. Phys. Rev. B: Condens. Matter Mater. Phys. 1994, 49, 776-785. 
(29) Lin, K.; Zhou, Z.; Liu, L.; Ma, H.; Chen, J.; Deng, J.; Sun, J.; You, L.; Kasai, H.; Kato, K.; Takata, M.; Xing, X., Unusual Strong Incommensurate Modulation in a TungstenBronze-Type Relaxor PbBiNb5O15. J. Am. Chem. Soc. 2015, 137, 13468-71.

(30) Ma, H.; Lin, K.; Liu, L.; Yang, B.; Rong, Y.; Chen, J.; Deng, J.; Kawaguchi, S.; Kato, K.; Xing, X., Structure and electrical properties of tetragonal tungsten bronze Ba2CeFeNb4O15. RSC Adv. 2015, 5, 76957-76962.

(31) Stennett, M. C.; Reaney, I. M.; Miles, G. C.; Woodward, D. I.; West, A. R.; Kirk, C. A.; Levin, I., Dielectric and structural studies of Ba2MTi2Nb3O15 where $\mathrm{M}=\mathrm{Bi3}+, \mathrm{La} 3+, \mathrm{Nd} 3+, \mathrm{Sm} 3+, \mathrm{Gd} 3+)$ tetragonal tungsten bronze-structured ceramics. J. Appl. Phys. 2007, 101, 104114.

(32) Ke, S.; Fan, H.; Huang, H.; Chan, H. L. W.; Yu, S., Dielectric, ferroelectric properties, and grain growth of CaxBa1-xNb2O6 ceramics with tungsten-bronzes structure. $J$. Appl. Phys. 2008, 104, 024101.

(33) Albino, M.; Veber, P.; Pechev, S.; Labrugère, C.; Velázquez, M.; Maglione, M.; Josse, M., Growth and characterization of Ba2LnFeNb4O 15 ( $\mathrm{Ln}=\mathrm{Pr}, \mathrm{Nd}, \mathrm{Sm}$, Eu) relaxor single crystals. Cryst. Growth Des. 2014, 14, 500-512.

(34) Huang, W. H.; Viehland, D.; Neurgaonkar, R. R., Anisotropic Glasslike Characteristics of Strontium Barium Niobate Relaxors. J. Appl. Phys. 1994, 76, 490-496.

(35) Schefer, J.; Schaniel, D.; Petříček, V.; Woike, T.; Cousson, A.; Wöhlecke, M., Reducing the positional modulation of NbO6-octahedra in $\mathrm{Sr} \times \mathrm{Ba} 1-\mathrm{xNb} 2 \mathrm{O} 6$ by increasing the barium content: A single crystal neutron diffraction study at ambient temperature for $\mathrm{x}=$ 0.61 and $\mathrm{x}=0.34$. Z. Kristallogr. 2008, 223, 399-407.

(36) Randall, C. A.; Guo, R.; Bhalla, A. S.; Cross, L. E., Microstructure-Property Relations in Tungsten Bronze Lead Barium Niobate, Pb1-Xbaxnb2o6. J. Mater. Res. 1991, 6, 17201728.

(37) Zhu, X. L.; Li, K.; Chen, X. M.; Green, D. J., Ferroelectric Transition and LowTemperature Dielectric Relaxations in Filled Tungsten Bronzes. J. Am. Ceram. Soc. 2014, 97, 329-338.

(38) Labbe, P.; Leligny, H.; Raveau, B.; Schneck, J.; Toledano, J. C., X-ray structural determination of the quasi-commensurate phase of barium sodium niobate. J. Phys.: Condens. Matter 1990, 2, 25-43.

(39) Zhu, X. L.; Wu, S. Y.; Chen, X. M., Dielectric anomalies in (BaxSr1$\mathrm{x})(4) \mathrm{Nd} 2 \mathrm{Ti} 4 \mathrm{Nb} 6 \mathrm{O} 30$ ceramics with various radius differences between A1-and A2-site ions. Appl. Phys. Lett. 2007, 91, 162906.

(40) Gardner, J.; Morrison, F. D., A-site size effect in a family of unfilled ferroelectric tetragonal tungsten bronzes: Ba4R0.67Nb10O30 ( $\mathrm{R}=\mathrm{La}, \mathrm{Nd}, \mathrm{Sm}, \mathrm{Gd}$, Dy and Y). Dalton Trans. 2014, 43, 11687-11695.

(41) Thompson, S. P.; Parker, J. E.; Potter, J.; Hill, T. P.; Birt, A.; Cobb, T. M.; Yuan, F.; Tang, C. C., Beamline $\mathrm{I11}$ at Diamond: a new instrument for high resolution powder diffraction. Rev. Sci. Instrum. 2009, 80, 075107.

(42) Hannon, A. C., Results on disordered materials from the GEneral Materials diffractometer, GEM, at ISIS. Nucl. Instrum. Methods Phys. Res., Sect. A 2005, 551, 88-107.

(43) Rotaru, A.; Arnold, D. C.; Daoud-Aladine, A.; Morrison, F. D., Origin and stability of the dipolar response in a family of tetragonal tungsten bronze relaxors. Phys. Rev. B Condens. Matter Mater. Phys. 2011, 83.

(44) Rotaru, A.; Miller, A. J.; Arnold, D. C.; Morrison, F. D., Towards novel multiferroic and magnetoelectric materials: dipole stability in tetragonal tungsten bronzes. Philos. Trans. R. Soc., A 2014, 372, 20120451.

(45) Morrison, F. D.; Sinclair, D. C.; West, A. R., Electrical and structural characteristics of lanthanum-doped barium titanate ceramics. J. Appl. Phys. 1999, 86, 6355-6366. 
(46) Bokov, A. A.; Maglione, M.; Ye, Z. G., Quasi-ferroelectric state in Ba(Ti 1- x Zr x )O 3 relaxor: dielectric spectroscopy evidence. J. Phys.: Condens. Matter 2007, 19, 092001.

(47) Bonneau, P.; Garnier, P.; Calvarin, G.; Husson, E.; Gavarri, J. R.; Hewat, A. W.; Morell, A., X-ray and neutron diffraction studies of the diffuse phase transition in $\mathrm{PbMg} 1$ 3Nb2 $3 \mathrm{O} 3$ ceramics. J. Solid State Chem. 1991, 91, 350-361.

(48) Hornebecq, V.; Elissalde, C.; Gravereau, P.; Lebraud, E.; Ravez, J., Effect of cationic substitutions on ferroelectric properties of Pb5Ta10O30: Generation of the relaxor behavior. J. Solid State Chem. 2001, 157, 261-273.

(49) Gorev, M. V.; Flerov, I. N.; Tressaud, A.; Molokeev, M. S.; Kartashev, A. V.; Pogoreltsev, E. I.; Bayukov, O. A., Phase transitions in fluoride KFe2F6 with tetragonal tungsten bronze structure. J. Fluorine Chem. 2014, 168, 204-211.

(50) Shirane, G., Ferroelectricity and Antiferroelectricity in Ceramic Pbzro3 Containing Ba or Sr. Phys. Rev. 1952, 86, 219-227.

(51) Shirane, G.; Sawaguchi, E.; Takagi, Y., Dielectric Properties of Lead Zirconate. Phys. Rev. 1951, 84, 476-481.

(52) Jin, L.; Li, F.; Zhang, S., Decoding the Fingerprint of Ferroelectric Loops: Comprehension of the Material Properties and Structures. J. Am. Ceram. Soc. 2014, 97, 1-27.

(53) Ren, X., Large electric-field-induced strain in ferroelectric crystals by point-defectmediated reversible domain switching. Nat. Mater. 2004, 3, 91-4.

(54) Merz, W. J., Double hysteresis loop of BaTiO3 at the curie point. Phys. Rev. 1953, 91, 513-517.

(55) Granzow, T.; Suvaci, E.; Kungl, H.; Hoffmann, M. J., Deaging of heat-treated irondoped lead zirconate titanate ceramics. Appl. Phys. Lett. 2006, 89, 262908.

(56) Tan, Q.; Li, J.; Viehland, D., Role of lower valent substituent-oxygen vacancy complexes in polarization pinning in potassium-modified lead zirconate titanate. Appl. Phys. Lett. 1999, 75, 418-420.

(57) Koval, V.; Viola, G.; Tan, Y.; Peláiz-Barranco (Editor), A., Biasing Effects in Ferroic Materials, Ferroelectric Materials - Synthesis and Characterization. 2015.

(58) Zhou, W.; Jefferson, D. A.; Thomas, J. M., Defect Fluorite Structures Containing BiFormulaOFormula: the System BiFormulaOFormula-NbFormulaOFormula. Proc. R. Soc. London, Ser. A 1986, 406, 173-182.

(59) Tang, D.; Zhou, W., An electron diffraction study of the type II Bi2-xNbxO3+x solid solution. J. Solid State Chem. 1995, 119, 311-318.

(60) Ruiz-Morales, J. C.; Canales-Vazquez, J.; Savaniu, C.; Marrero-Lopez, D.; Zhou, W.; Irvine, J. T., Disruption of extended defects in solid oxide fuel cell anodes for methane oxidation. Nature 2006, 439, 568-71.

(61) Zhang, Y.; Su, Z.; Azad, A. K.; Zhou, W.; Irvine, J. T. S., Directly Imaging Interstitial Oxygen in Silicate Apatite. Adv. Energy Mater. 2012, 2, 316-321.

(62) Reisinger, S. A.; Leblanc, M.; Mercier, A. M.; Tang, C. C.; Parker, J. E.; Morrison, F. D.; Lightfoot, P., Phase Separation and Phase Transitions in Multiferroic K0.58FeF3 with the Tetragonal Tungsten Bronze Structure. Chem. Mater. 2011, 23, 5440-5445.

(63) Kuang, X. J.; Pan, F. J.; Cao, J.; Liang, C. L.; Suchomel, M. R.; Porcher, F.; Allix, M., Defect Structure, Phase Separation, and Electrical Properties of Nonstoichiometric Tetragonal Tungsten Bronze Ba0.5-xTaO3-x. Inorg. Chem. 2013, 52, 13244-13252.

(64) Sugai, T., Ferroelectric Crystal of Ba2Bi1/3 Nb5O15. Jpn. J. Appl. Phys., Part 1 1987, 26, 778-779.

(65) Castel, E.; Josse, M.; Michau, D.; Maglione, M., Flexible relaxor materials: $\mathrm{Ba}(2) \mathrm{Pr}(\mathrm{x}) \mathrm{Nd}(1-\mathrm{x}) \mathrm{FeNb}(4) \mathrm{O}(15)$ tetragonal tungsten bronze solid solution. J Phys Condens Matter 2009, 21, 452201. 
(66) Venet, M.; M'Peko, J. C.; Zabotto, F. L.; Guerrero, F.; Garcia, D.; Eiras, J. A., Dynamics of normal to diffuse and relaxor phase transition in lead metaniobate-based ferroelectric ceramics. Appl. Phys. Lett. 2009, 94.

(67) Prades, M.; Maso, N.; Beltran, H.; Cordoncillo, E.; West, A. R., Synthesis, structural characterization, and electrical properties of new oxygen-deficient tetragonal tungsten bronzes Ba2NdTi(2+x) $\mathrm{Nb}(3-\mathrm{x}) \mathrm{O}(15-\mathrm{x} / 2)$. Inorg. Chem. 2013, 52, 1729-1736.

(68) Kinka, M.; Gabrielaitis, D.; Albino, M.; Josse, M.; Palaimiene, E.; Grigalaitis, R.; Maglione, M.; Banys, J., Investigation of Dielectric Relaxation Processes in Ba2NdFeNb4xTaxO15 Ceramics. Ferroelectrics 2015, 485, 101-109.

(69) Josse, M.; Heijboer, P.; Albino, M.; Molinari, F.; Porcher, F.; Decourt, R.; Michau, D.; Lebraud, E.; Veber, P.; Velazquez, M.; Maglione, M., Original crystal-chemical behaviors in $(\mathrm{Ba}, \mathrm{Sr}) 2 \mathrm{Ln}(\mathrm{Fe}, \mathrm{Nb}, \mathrm{Ta}) 5 \mathrm{O} 15$ tetragonal tungsten bronze: Anion-driven properties evidenced by cationic substitutions. Cryst. Growth Des. 2014, 14, 5428-5435.

(70) Venet, M.; Zabotto, F. L.; Garcia, J. E.; Ochoa, D. A.; Garcia, D.; Eiras, J. A.; M'Peko, J. C., La3+-induced (micro)structural changes and origin of the relaxor-like phase transition in ferroelectric lead barium niobate electroceramics. J. Mater. Sci. 2014, 49, 48254832 .

(71) Ko, J. H.; Jiang, F.; Kojima, S., Dielectric and pyroelectric properties of strontium barium niobate at low temperatures. Ferroelectrics 2002, 270, 1395-1400.

(72) Ravez, J.; Perron, A.; Chaminade, J. P.; Hagenmuller, P.; Rivoallan, L., Etude des propriétés cristallographiques, diélectriques et d'optique non linéaire de quelques nouvelles phases de composition BaxLi5-2xNb5(1-y)Ta5yO15 et de structure bronzes oxygénés de tungstène quadratiques. J. Solid State Chem. 1974, 10, 274-281.

(73) Amorin, H.; Perez, J.; Fundora, A.; Portelles, J.; Guerrero, F.; Soares, M. R.; Martinez, E.; Siqueiros, J. M., Evidence of short-range antiferroelectric local states in lanthanum- and titanium-modified $\mathrm{Sr} 0.3 \mathrm{Ba} 0.7 \mathrm{Nb} 2 \mathrm{O} 6$ ferroelectric ceramics. Appl. Phys. Lett. 2003, 83, 4390-4392.

(74) Gagou, Y.; Amira, Y.; Lukyanchuk, I.; Mezzane, D.; Courty, M.; Masquelier, C.; Yuzyuk, Y. I.; El Marssi, M., On the nature of phase transitions in the tetragonal tungsten bronze GdK2Nb5O15 ceramics. J. Appl. Phys. 2014, 115.

(75) Shikhova, V. A.; Shur, V. Y.; Pelegov, D. V.; Ivleva, L. I., Double Loops Formation in $\mathrm{Sr} 0.75 \mathrm{Ba} 0.25 \mathrm{Nb} 2 \mathrm{O} 6$ Single Crystals in Relaxor Phase. Ferroelectrics 2013, 443, 116-123.

(76) Jonker, G. H., Nature of Aging in Ferroelectric Ceramics. J. Am. Ceram. Soc. 1972, $55,57-58$.

(77) Trolliard, G.; Dorcet, V., Reinvestigation of Phase Transitions in Na0.5Bi0.5TiO3 by TEM. Part II: Second Order Orthorhombic to Tetragonal Phase Transition. Chem. Mater. 2008, 20, 5074-5082.

(78) Dorcet, V.; Trolliard, G.; Boullay, P., Reinvestigation of Phase Transitions in $\mathrm{Na} 0.5 \mathrm{Bi} 0.5 \mathrm{TiO} 3$ by TEM. Part I: First Order Rhombohedral to Orthorhombic Phase Transition. Chem. Mater. 2008, 20, 5061-5073.

(79) Isupov, V. A., Ferroelectric $\mathrm{Na} 0.5 \mathrm{Bi} 0.5 \mathrm{TiO} 3$ and $\mathrm{K} 0.5 \mathrm{Bi} 0.5 \mathrm{TiO} 3$ perovskites and their solid solutions. Ferroelectrics 2005, 315, 123-147.

(80) Levin, I.; Reaney, I. M.; Anton, E. M.; Jo, W.; Rödel, J.; Pokorny, J.; Schmitt, L. A.; Kleebe, H. J.; Hinterstein, M.; Jones, J. L., Local structure, pseudosymmetry, and phase transitions in Na1/2Bi1/2TiO3-K1/2Bi1/2TiO3ceramics. Phys. Rev. B Condens. Matter Mater. Phys. 2013, 87.

(81) Viola, G.; Ning, H.; Wei, X.; Deluca, M.; Adomkevicius, A.; Khaliq, J.; John Reece, M.; Yan, H., Dielectric relaxation, lattice dynamics and polarization mechanisms in Bi0.5Na0.5TiO3-based lead-free ceramics. J. Appl. Phys. 2013, 114, 014107. 
(82) Shi, J.; Fan, H.; Liu, X.; Bell, A. J., Large Electrostrictive Strain in (Bi0.5Na0.5)TiO3-BaTiO3-(Sr0.7Bi0.2)TiO3 Solid Solutions. J. Am. Ceram. Soc. 2014, 97, 848-853.

(83) Rödel, J.; Jo, W.; Seifert, K. T. P.; Anton, E. M.; Granzow, T.; Damjanovic, D., Perspective on the development of lead-free piezoceramics. J. Am. Ceram. Soc. 2009, 92, 1153-1177.

(84) Jo, W.; Schaab, S.; Sapper, E.; Schmitt, L. A.; Kleebe, H.-J.; Bell, A. J.; Rödel, J., On the phase identity and its thermal evolution of lead free (Bi1/2Na1/2)TiO3-6 mol\% BaTiO3. J. Appl. Phys. 2011, 110, 074106.

\section{Figure Captions}

Figure 1. Aristotype TTB structure $\left(P 4 / \mathrm{mbm}\right.$, solid line) viewed along $001_{\mathrm{TTB}}$ and related Bbm2 supercell $\left(2 \sqrt{2} a_{\mathrm{TTB}} \times \sqrt{ } 2 b_{\mathrm{TTB}} \times 2 c_{\mathrm{TTB}}\right)$ expansion (dashed line); the Ima 2 structure is generated by halving $a$ in the latter.

Figure 2. (a) Relative permittivity ( $\left.\varepsilon^{\prime}\right)$ and dielectric loss $\left(\varepsilon^{\prime \prime}\right)$ for $\mathrm{Ba}_{4} \mathrm{La}_{0.67} \mathrm{Nb}_{10} \mathrm{O}_{30}(x=0)$ at selected frequencies and (inset) high temperature dielectric loss peak (1 MHz); (b) Relative permittivity $\left(\varepsilon^{\prime}\right)$ and dielectric loss $\left(\varepsilon^{\prime \prime}\right)$ for $x=1\left(\mathrm{Ba}_{4} \mathrm{Nd}_{0.67} \mathrm{Nb}_{10} \mathrm{O}_{30}\right)$ at selected frequencies (arrows indicate increasing frequency).

Figure 3. (a) Rietveld refinement of PND data $(20 \mathrm{~K})$ for $x=0$ demonstrating $B b m 2$ superstructure and deficiency of simple tetragonal spacegroup $(P 4 b m)$ - observed data (black circles), calculated intensity, difference and background; (b) PND patterns at selected temperatures showing loss of superstructure reflections $(d=2.24,2.27$ and $2.48 \AA$, indicated by arrows) at elevated temperatures; (c) lattice parameters (superstructure parameters reduced to aristotype cell) and (d) volume and orthorhombicity as a function of temperature (from synchrotron PXRD data). Note that for all data points of (c) and (d) the calculated uncertainty is smaller than the plotted symbol.

Figure 4. SAED patterns from the $x=0$ sample, indexed using the Bbm2 supercell, (a) showing a commensurate superstructure when viewing down the [011] axis and (b) showing diffuse diffraction spots along the [110] direction, and (c) showing diffuse diffraction spots along the [250] direction.

Figure 5. Polarization-electric field (P-E) loops and associated current (I-E) data for $x=0$ at selected temperatures ('backswitching' event on unloading indicated by arrows).

Figure 6. Temperature dependence of (a) coercive $\left(+E_{C}\right)$ and depolarization $\left(+E_{D}\right)$ field, and (b) remanent polarization and maximum polarization. 
Figure 7. HRTEM image and the corresponding SAED pattern from the $\mathrm{x}=1$ sample. The inset is an enlarged image showing dark spot contrast with local atomic dislocation. The SAED pattern is indexed onto the TTB basic unit cell. The inter-spot distances, $a$ and $b$, are not equal, indicating an incommensurate structure modulation.

Figure 8. Relative permittivity $\left(\varepsilon^{\prime}\right)$ and dielectric loss $\left(\varepsilon^{\prime \prime}\right)$ for $\mathrm{Ba}_{4}\left(\mathrm{La}_{1-\mathrm{x}} \mathrm{Nd}_{\mathrm{x}}\right)_{0.67} \mathrm{Nb}_{10} \mathrm{O}_{30},(1$ MHz data). 


\section{Figures}

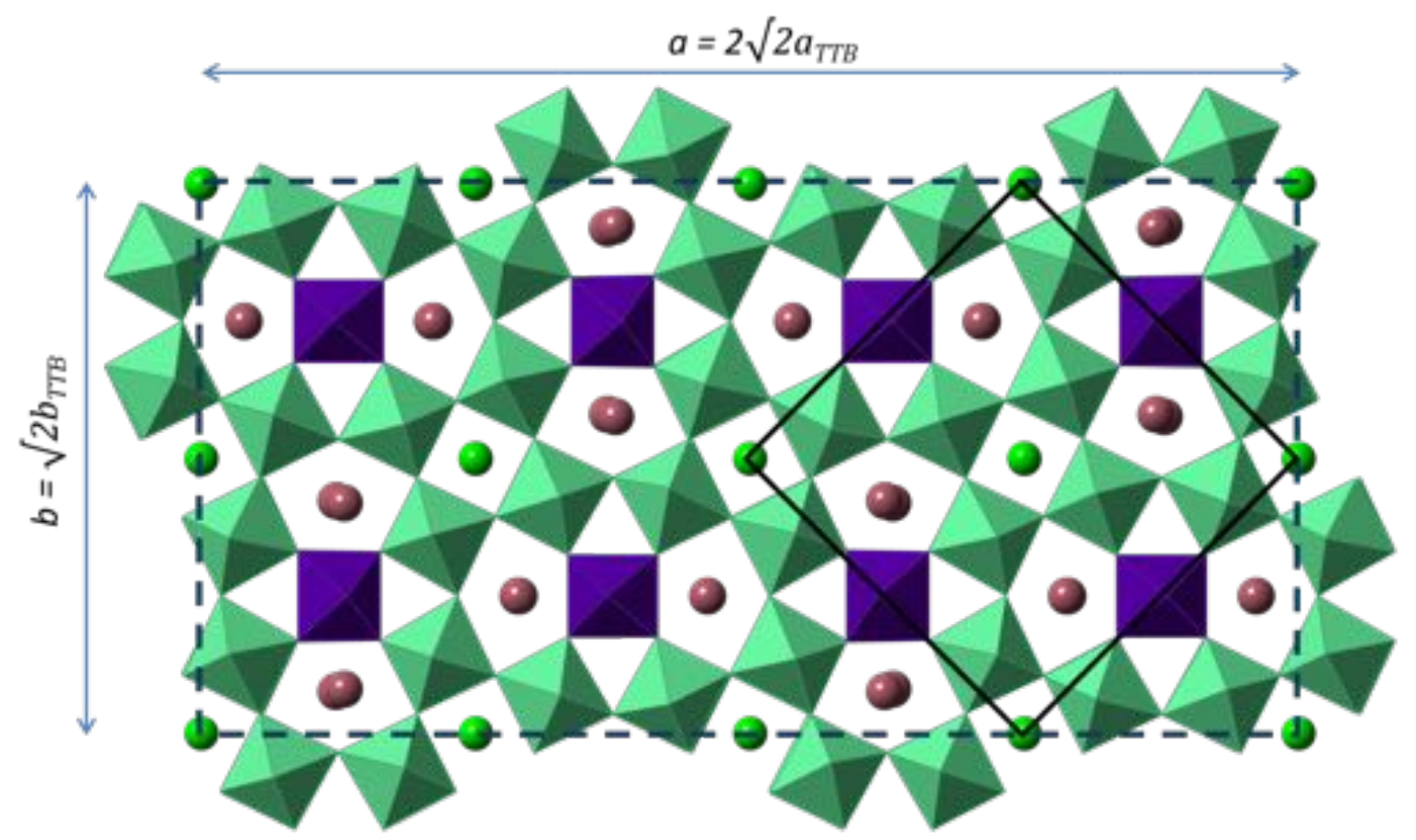

Figure 1. Aristotype TTB structure $(P 4 / \mathrm{mbm} \text {, solid line) viewed along [001] }]_{\mathrm{TTB}}$ and related $B b m 2$ supercell $\left(2 \sqrt{2} a_{\mathrm{TTB}} \times \sqrt{ } 2 a_{\mathrm{TTB}} \times 2 c_{\mathrm{TTB}}\right)$ expansion (dashed line); the Ima2 structure is generated by halving $a$ in the latter. 
(a)

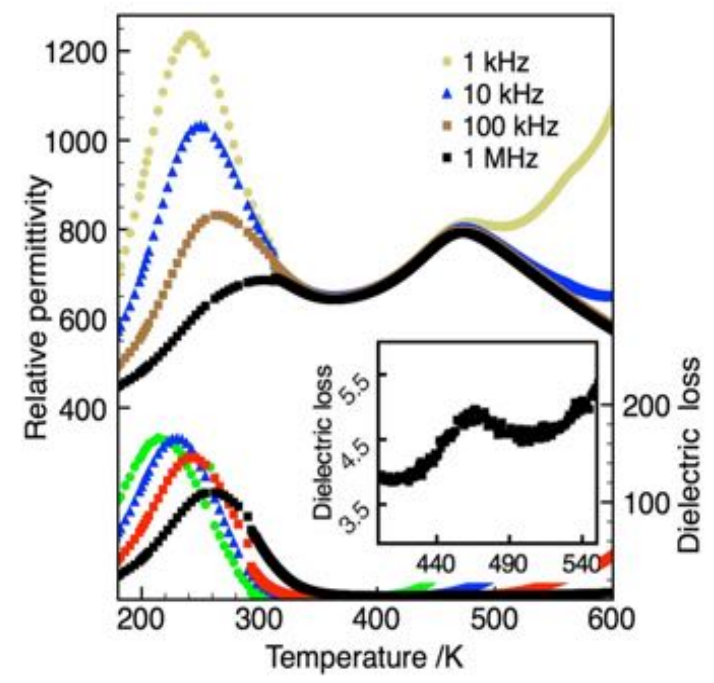

(b)

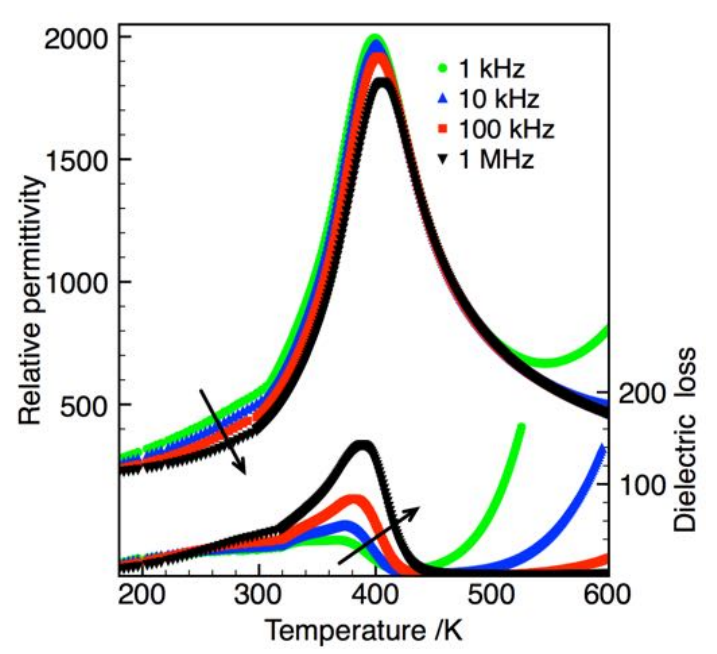

Figure 2. (a) Relative permittivity ( $\left.\varepsilon^{\prime}\right)$ and dielectric loss $\left(\varepsilon^{\prime \prime}\right)$ for $\mathrm{Ba}_{4} \mathrm{La}_{0.67} \mathrm{Nb}_{10} \mathrm{O}_{30}(x=0)$ at selected frequencies and (inset) high temperature dielectric loss peak (1 MHz); (b) Relative permittivity $\left(\varepsilon^{\prime}\right)$ and dielectric loss $\left(\varepsilon^{\prime \prime}\right)$ for $x=1\left(\mathrm{Ba}_{4} \mathrm{Nd}_{0.67} \mathrm{Nb}_{10} \mathrm{O}_{30}\right)$ at selected frequencies (arrows indicate increasing frequency). 
(a)

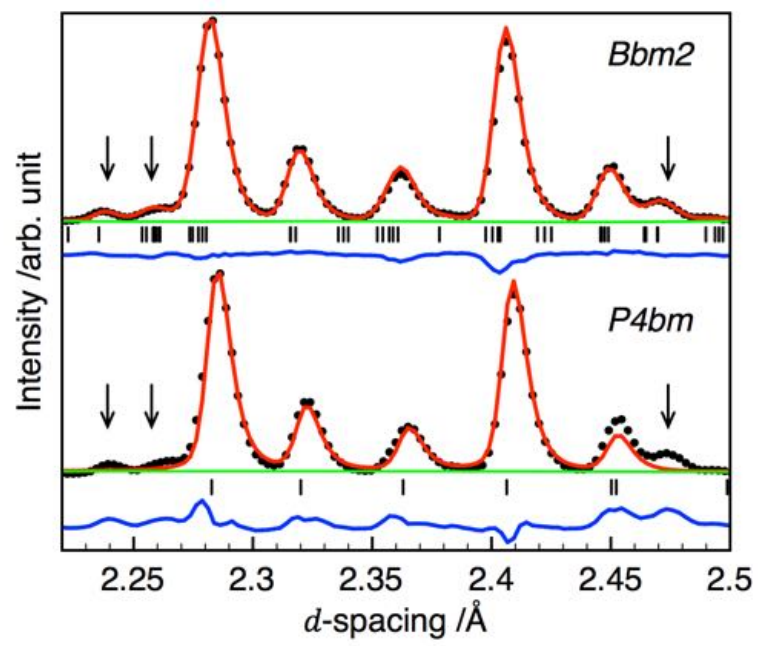

(c)

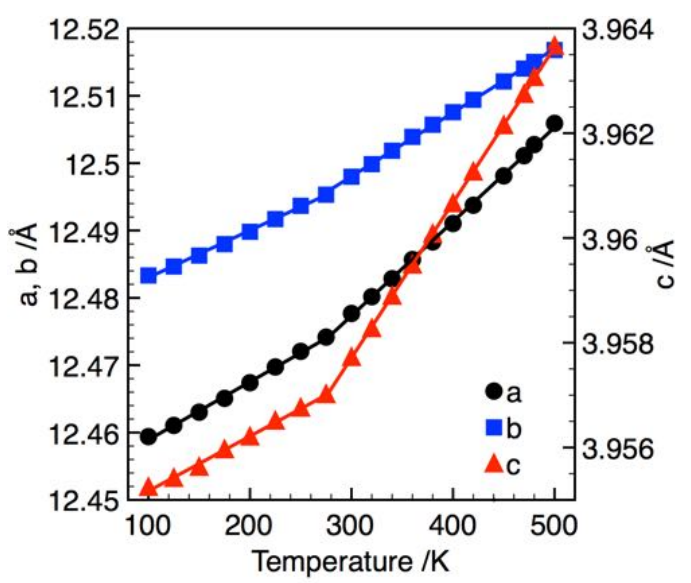

(b)

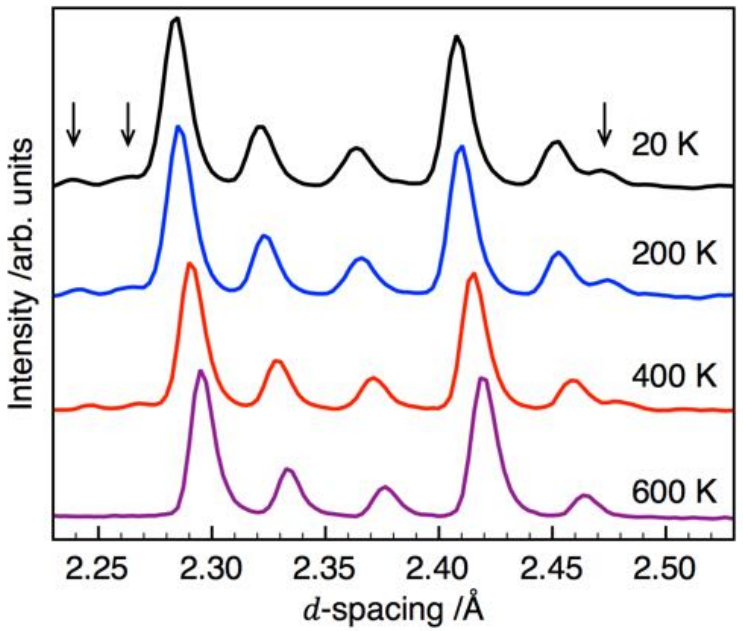

(d)

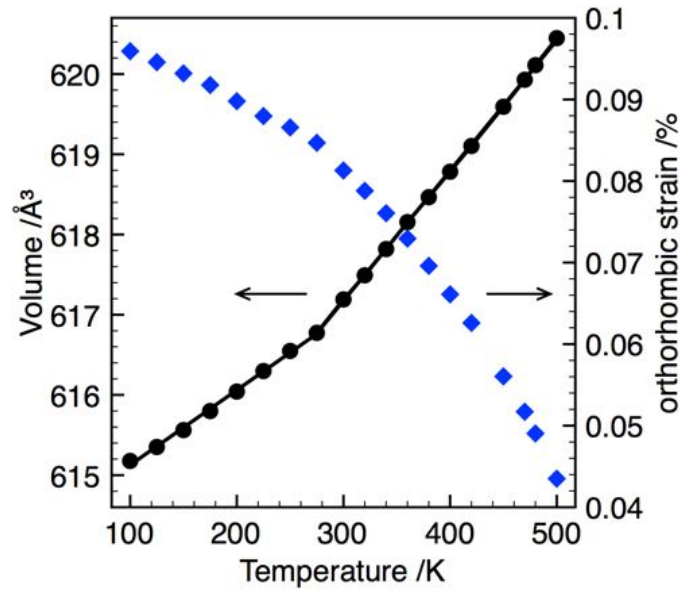

Figure 3. (a) Rietveld refinement of PND data $(20 \mathrm{~K})$ for $x=0$ demonstrating $B b m 2$ superstructure and deficiency of simple tetragonal spacegroup $(\mathrm{P} 4 \mathrm{bm})$ - observed data (black circles), calculated intensity, difference and background; (b) PND patterns at selected temperatures showing loss of superstructure reflections $(d=2.24,2.27$ and $2.48 \AA$, indicated by arrows) at elevated temperatures; (c) lattice parameters (superstructure parameters reduced to aristotype cell) and (d) volume and orthorhombicity as a function of temperature (from synchrotron PXRD data). Note that for all data points of (c) and (d) the calculated uncertainty is smaller than the plotted symbol. 

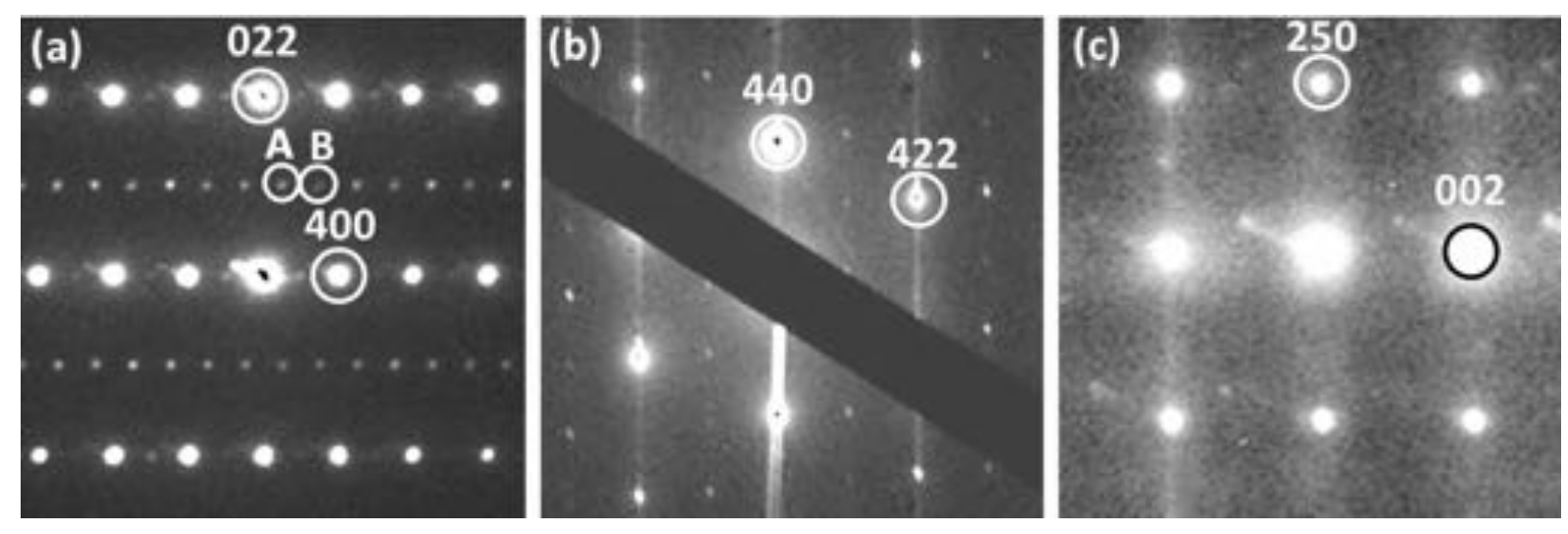

Figure 4. SAED patterns from the $x=0$ sample, indexed using the $B b m 2$ supercell, (a) showing a commensurate superstructure when viewing down the [01 $\overline{1}]$ axis and (b) showing diffuse diffraction spots along the [110] direction, and (c) showing diffuse diffraction spots along the [250] direction. 

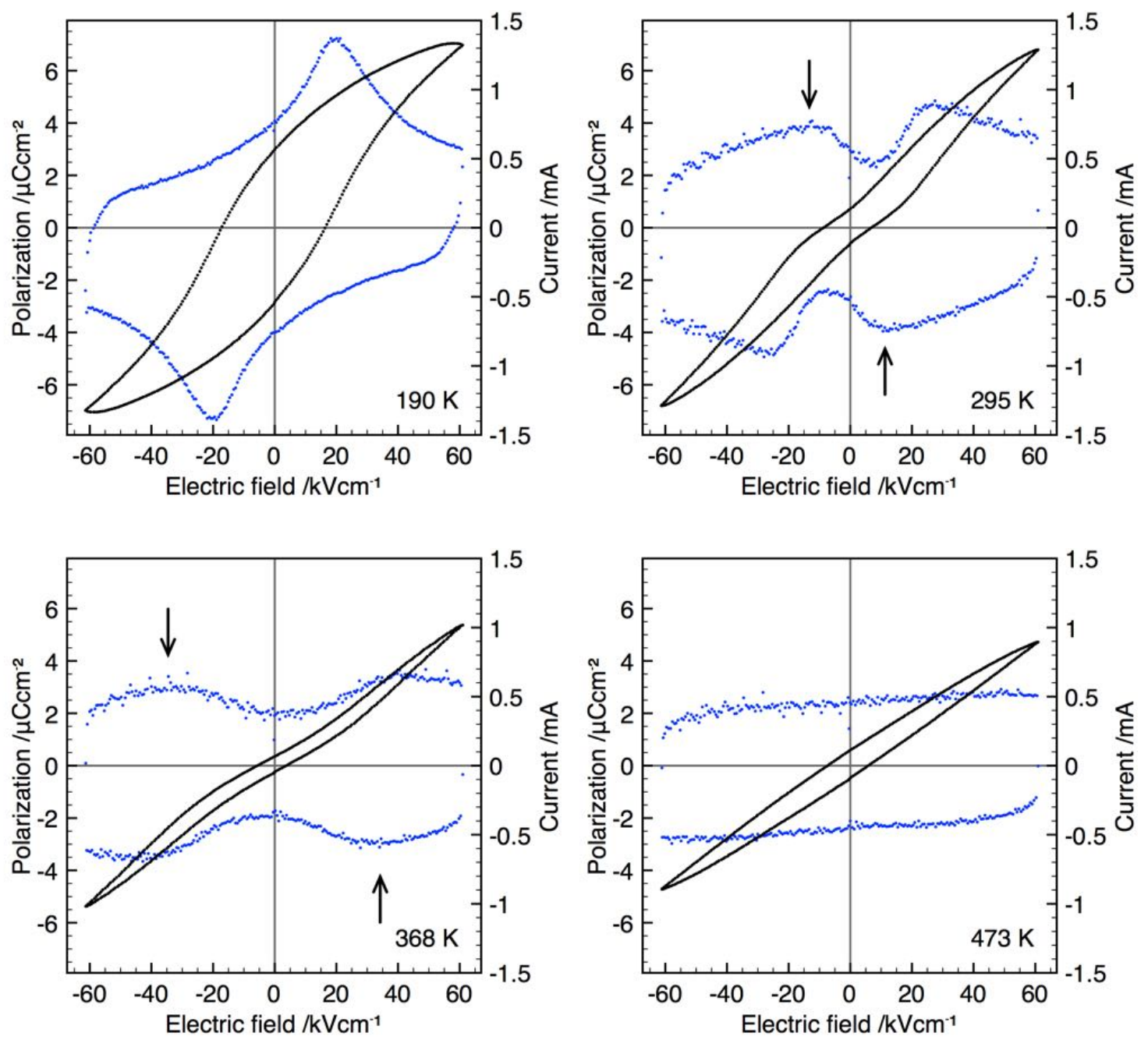

Figure 5. Polarization-electric field (P-E) loops and associated current-field (I-E) data for $x=$ 0 at selected temperatures ('backswitching' event on unloading indicated by arrows). 
(a)

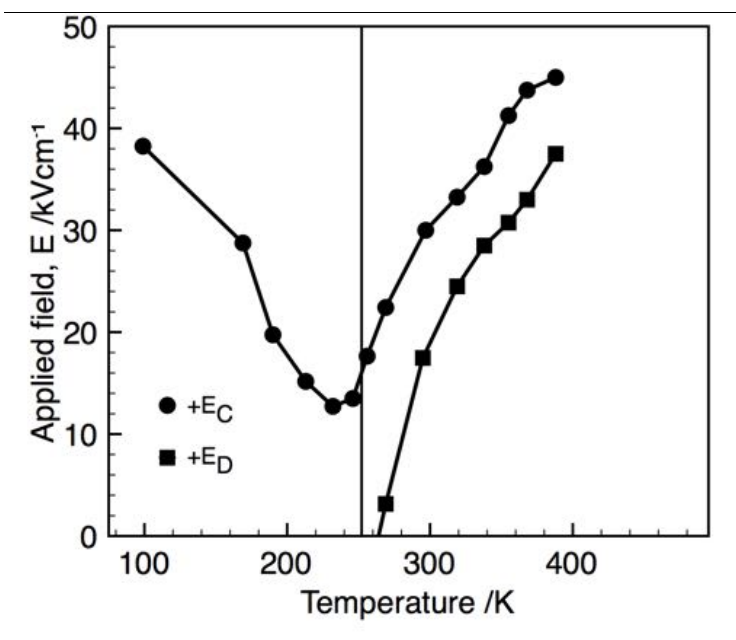

(b)

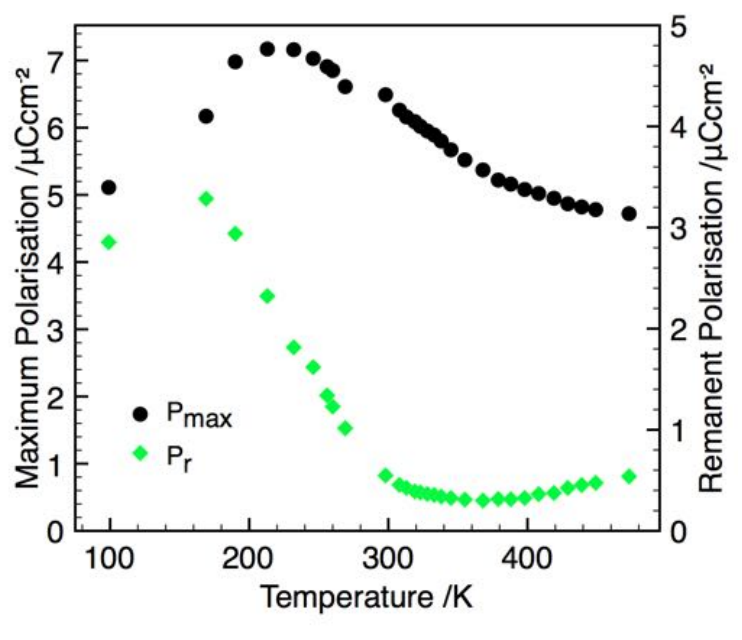

Figure 6. Temperature dependence of (a) coercive $\left(+E_{C}\right)$ and depolarization $\left(+E_{D}\right)$ field, and (b) remanent polarization and maximum polarization. 


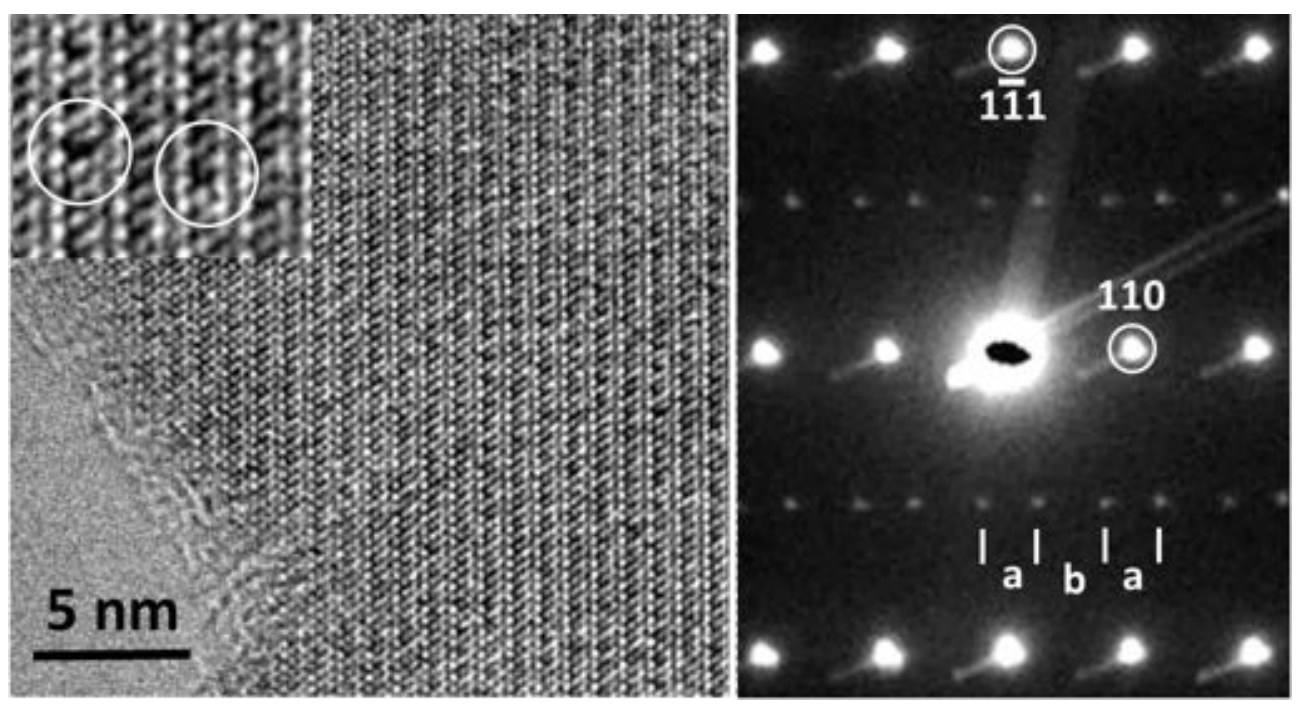

Figure 7. HRTEM image and the corresponding SAED pattern from the $\mathrm{x}=1$ sample. The inset is an enlarged image showing dark spot contrast with local atomic dislocation. The SAED pattern is indexed onto the TTB basic unit cell. The inter-spot distances, $a$ and $b$, are not equal, indicating an incommensurate structure modulation. 


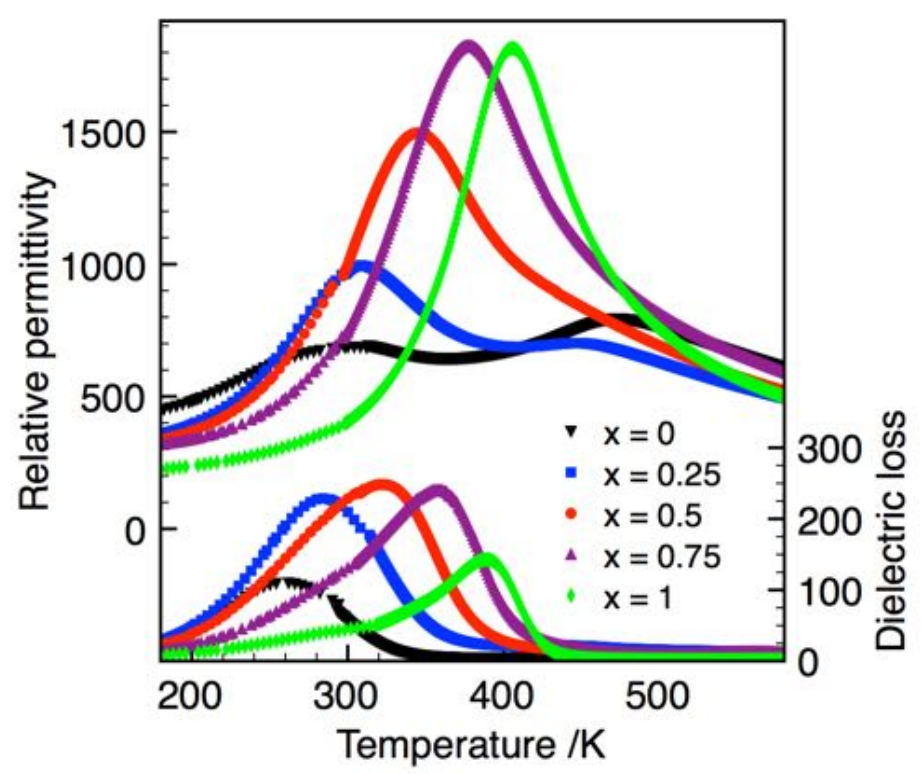

Figure 8. Relative permittivity $\left(\varepsilon^{\prime}\right)$ and dielectric loss $\left(\varepsilon^{\prime \prime}\right)$ for $\mathrm{Ba}_{4}\left(\mathrm{La}_{1-\mathrm{x}} \mathrm{Nd}_{\mathrm{x}}\right)_{0.67} \mathrm{Nb}_{10} \mathrm{O}_{30},(1$ $\mathrm{MHz}$ data). 


\section{Electronic Supporting Information}

\section{Relaxor-to-ferroelectric crossover and disruption of polar order in 'empty' tetragonal tungsten bronzes}

Jonathan Gardner ${ }^{1}$, Fengjiao $\mathrm{Yu}^{1}$, Chiu Tang ${ }^{2}$, Winfried Kockelmann ${ }^{3}$, Wuzong Zhou ${ }^{1}$, Finlay D. Morrison ${ }^{1, *}$

${ }^{1}$ EaStCHEM School of Chemistry, University of St Andrews, North Haugh, St Andrews, KY16 9ST, UK

${ }^{2}$ Diamond Light Source Ltd., Harwell Science and Innovation Campus, Didcot OX11 0DE, UK

${ }^{3}$ ISIS Facility, Rutherford Appleton Laboratory, Chilton, Didcot OX11 0QX, UK

\section{Structural}

Variable temperature PND data for $\mathrm{Ba}_{4} \mathrm{La}_{0.67} \mathrm{Nb}_{10} \mathrm{O}_{30}(x=0)$ collected on the GEM beamline at ISIS (as detailed in the main text). Rietveld refinements of data in polar space group Bbm2 demonstrate requirement of a supercell, Figure S1.

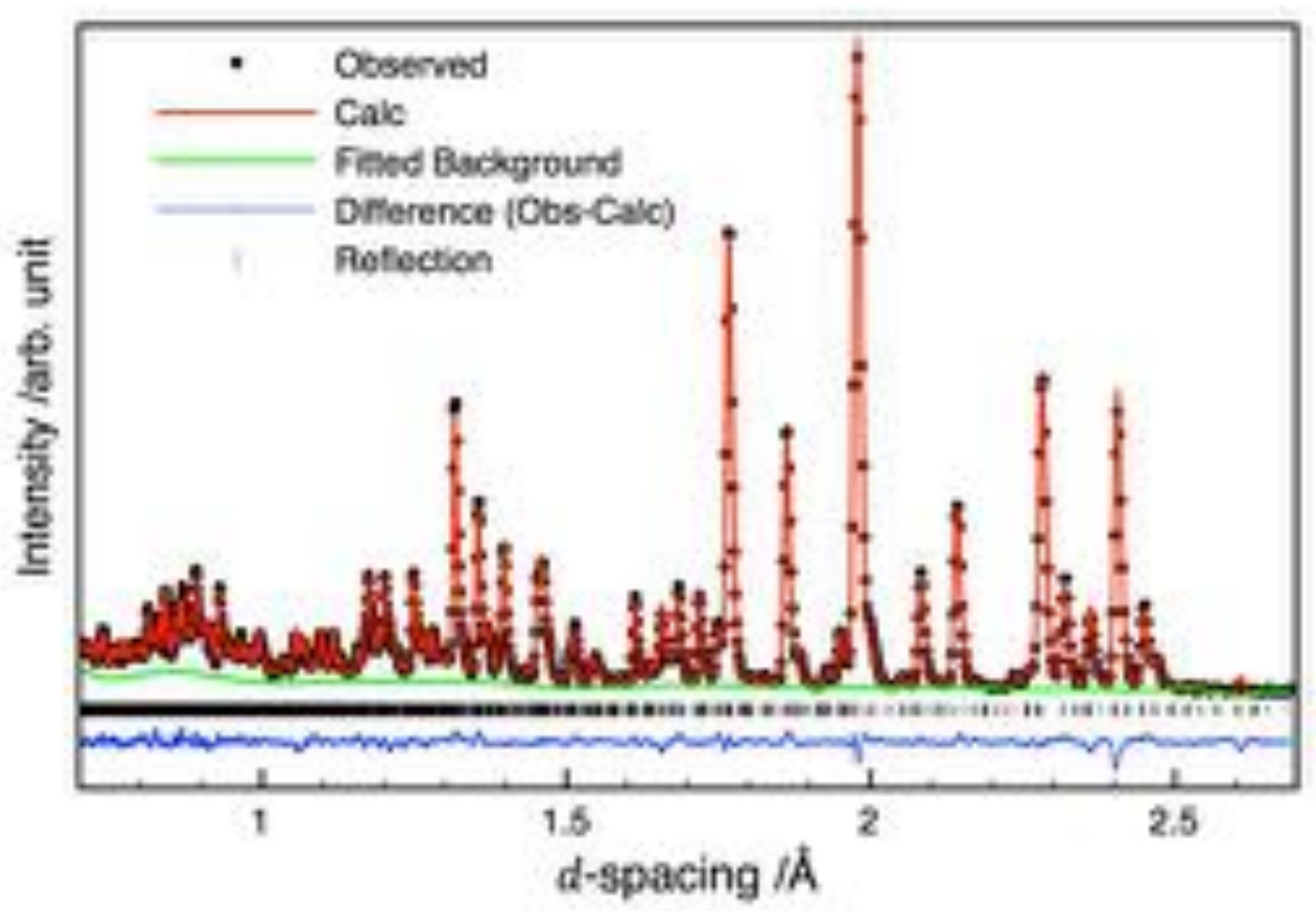

Figure S1: Rietveld refinement of PND data for $\mathrm{Ba}_{4} \mathrm{La}_{0.67} \mathrm{Nb}_{10} \mathrm{O}_{30}(x=0)$ at $20 \mathrm{~K}$ in space group Bbm2; including observed data, calculated profile, fitted background, difference plot and reflection markers. 
Table S1. Atomic positions of $\mathrm{Ba}_{4} \mathrm{La}_{0.67} \mathrm{Nb}_{10} \mathrm{O}_{30}(x=0)$ in space group $B b m 2$

\begin{tabular}{|c|c|c|c|c|}
\hline Site Label & Atom & $x$ & $y$ & $z$ \\
\hline A2_1 & $\mathrm{Ba}$ & 0.8774 & 0.4237 & 0.25 \\
\hline A2_2 & $\mathrm{Ba}$ & -0.1301 & 0.4192 & 0.7484 \\
\hline A2 3 & $\mathrm{Ba}$ & 0.0387 & 0.25 & 0.2464 \\
\hline A2_4 & $\mathrm{Ba}$ & 0.0388 & 0.25 & 0.75 \\
\hline A2_5 & $\mathrm{Ba}$ & 0.2883 & 0.75 & 0.2485 \\
\hline A2_6 & $\mathrm{Ba}$ & 0.2895 & 0.75 & 0.7491 \\
\hline A1_1 & $\mathrm{La}$ & 0 & 0.5 & 0.2496 \\
\hline A1_2 & $\mathrm{La}$ & 0 & 0.5 & 0.752 \\
\hline A1_3 & $\mathrm{La}$ & 0.2499 & 0.0005 & 0.2456 \\
\hline $\mathrm{Nb} 1$ & $\mathrm{Nb}$ & 0.8757 & 0.25 & 0.0093 \\
\hline $\mathrm{Nb} 2$ & $\mathrm{Nb}$ & -0.1252 & 0.25 & 0.507 \\
\hline $\mathrm{Nb} 3$ & $\mathrm{Nb}$ & -0.1253 & 0.75 & 0.0059 \\
\hline $\mathrm{Nb} 4$ & $\mathrm{Nb}$ & 0.8753 & 0.75 & 0.5058 \\
\hline $\mathrm{Nb5}$ & $\mathrm{Nb}$ & 0.965 & 0.3586 & 0.005 \\
\hline Nb6 & $\mathrm{Nb}$ & 0.9667 & 0.3584 & 0.5065 \\
\hline $\mathrm{Nb} 7$ & $\mathrm{Nb}$ & 0.2168 & 0.8582 & 0.0051 \\
\hline $\mathrm{Nb} 8$ & $\mathrm{Nb}$ & 0.2155 & 0.858 & 0.5038 \\
\hline $\mathrm{Nb} 9$ & $\mathrm{Nb}$ & 0.071 & 0.4334 & 0.0061 \\
\hline $\mathrm{Nb} 10$ & $\mathrm{Nb}$ & 0.0703 & 0.4307 & 0.5068 \\
\hline $\mathrm{Nb} 11$ & $\mathrm{Nb}$ & 0.3207 & 0.9322 & 0.0025 \\
\hline $\mathrm{Nb} 12$ & $\mathrm{Nb}$ & 0.3215 & 0.9319 & 0.5049 \\
\hline $\mathrm{O} 1$ & $\mathrm{O}$ & 0.912 & 0.3302 & -0.0451 \\
\hline $\mathrm{O} 2$ & $\mathrm{O}$ & 0.9122 & 0.3338 & 0.505 \\
\hline $\mathrm{O} 3$ & $\mathrm{O}$ & 0.1627 & 0.8312 & 0.0055 \\
\hline $\mathrm{O} 4$ & $\mathrm{O}$ & 0.163 & 0.8309 & 0.4546 \\
\hline O5 & $\mathrm{O}$ & 0.9827 & 0.25 & -0.0098 \\
\hline O6 & $\mathrm{O}$ & 0.9819 & 0.25 & 0.4631 \\
\hline $\mathrm{O} 7$ & $\mathrm{O}$ & 0.2333 & 0.75 & -0.0165 \\
\hline O8 & $\mathrm{O}$ & 0.2333 & 0.75 & 0.5001 \\
\hline O9 & $\mathrm{O}$ & 0.8752 & 0.5334 & -0.0319 \\
\hline O10 & $\mathrm{O}$ & 0.8754 & 0.535 & 0.5038 \\
\hline O11 & $\mathrm{O}$ & 0.9473 & 0.4641 & -0.0451 \\
\hline $\mathrm{O} 12$ & $\mathrm{O}$ & 0.9438 & 0.4657 & 0.4972 \\
\hline O13 & $\mathrm{O}$ & 0.1968 & 0.964 & -0.0128 \\
\hline O14 & $\mathrm{O}$ & 0.1965 & 0.9638 & 0.4651 \\
\hline O15 & $\mathrm{O}$ & 0.983 & 0.6046 & 0.0091 \\
\hline O16 & $\mathrm{O}$ & 0.9819 & 0.6092 & 0.448 \\
\hline O17 & $\mathrm{O}$ & 0.232 & 0.1037 & 0.0062 \\
\hline O18 & $\mathrm{O}$ & 0.2322 & 0.1062 & 0.4516 \\
\hline O19 & $\mathrm{O}$ & 0.916 & 0.6747 & -0.0037 \\
\hline $\mathrm{O} 20$ & $\mathrm{O}$ & 0.9146 & 0.6749 & 0.4676 \\
\hline $\mathrm{O} 21$ & $\mathrm{O}$ & 0.1657 & 0.1747 & -0.0348 \\
\hline $\mathrm{O} 22$ & $\mathrm{O}$ & 0.1661 & 0.1762 & 0.4881 \\
\hline $\mathrm{O} 23$ & $\mathrm{O}$ & 0.9586 & 0.3646 & 0.2329 \\
\hline $\mathrm{O} 24$ & $\mathrm{O}$ & 0.2098 & 0.8616 & 0.7343 \\
\hline $\mathrm{O} 25$ & $\mathrm{O}$ & 0.9753 & 0.3553 & 0.7329 \\
\hline $\mathrm{O} 26$ & $\mathrm{O}$ & 0.2248 & 0.8579 & 0.2334 \\
\hline $\mathrm{O} 27$ & $\mathrm{O}$ & 0.9263 & 0.5528 & 0.231 \\
\hline $\mathrm{O} 28$ & $\mathrm{O}$ & 0.1734 & 0.0684 & 0.2359 \\
\hline O29 & $\mathrm{O}$ & 0.9348 & 0.5802 & 0.7331 \\
\hline $\mathrm{O} 30$ & $\mathrm{O}$ & 0.1847 & 0.0626 & 0.7322 \\
\hline O31 & $\mathrm{O}$ & 0.8835 & 0.25 & 0.2328 \\
\hline O32 & $\mathrm{O}$ & -0.1328 & 0.25 & 0.7347 \\
\hline $\mathrm{O} 33$ & $\mathrm{O}$ & 0.1298 & 0.25 & 0.2359 \\
\hline O34 & $\mathrm{O}$ & 0.1199 & 0.25 & 0.7356 \\
\hline
\end{tabular}


Due to the size of the structure and presence of 55 crystallographically distinct atoms it was not possible to reliably refine atom positions and individual isotropic displacement parameters as the variables are highly correlated. Atomic coordinates listed in Table S1 are based on those obtained by Labbé and co-workers (Journal of Physics-Condensed Matter 1989, 2, 25).

Table S2. Refined lattice parameters and refinement quality parameters from PND data

\begin{tabular}{ccccccc}
\hline Temp / K & $\boldsymbol{a} / \boldsymbol{\AA}$ & $\boldsymbol{b} / \boldsymbol{\AA}$ & $\boldsymbol{c} / \boldsymbol{\AA}$ & $\boldsymbol{\chi}^{\mathbf{2}}$ & $\mathbf{w R}_{\mathbf{p}} / \mathbf{\%}$ & $\mathbf{R}\left(\mathbf{F}^{\mathbf{2}} \mathbf{/} \mathbf{\%}\right.$ \\
\hline 20 & $35.2649(30)$ & $17.6567(15)$ & $7.90895(19)$ & 9.449 & 4.37 & 4.24 \\
50 & $35.2656(30)$ & $17.6573(15)$ & $7.90899(19)$ & 9.843 & 4.38 & 4.31 \\
100 & $35.2764(30)$ & $17.6610(14)$ & $7.91062(18)$ & 9.299 & 4.25 & 4.19 \\
150 & $35.2851(31)$ & $17.6644(15)$ & $7.91206(18)$ & 8.904 & 4.15 & 4.01 \\
200 & $35.2952(31)$ & $17.6689(15)$ & $7.91356(18)$ & 8.88 & 4.15 & 4.28 \\
250 & $35.3085(32)$ & $17.6741(16)$ & $7.91528(18)$ & 8.558 & 4.07 & 4.25 \\
300 & $35.3211(34)$ & $17.6806(16)$ & $7.91695(19)$ & 9.797 & 4.37 & 5.04 \\
298 & $35.3223(34)$ & $17.6814(16)$ & $7.91664(19)$ & 8.879 & 4.48 & 5.36 \\
340 & $35.334(3)$ & $17.6859(17)$ & $7.91842(20)$ & 9.404 & 4.62 & 5.74 \\
380 & $35.348(4)$ & $17.6921(19)$ & $7.92076(21)$ & 10.38 & 4.85 & 5.87 \\
420 & $35.365(4)$ & $17.6978(20)$ & $7.92348(21)$ & 10.41 & 4.86 & 6.18 \\
450 & $35.378(5)$ & $17.7022(23)$ & $7.92545(22)$ & 11.9 & 5.17 & 6.4 \\
470 & $35.388(5)$ & $17.7058(26)$ & $7.92729(25)$ & 14.09 & 5.63 & 7.08 \\
480 & $35.394(6)$ & $17.7078(28)$ & $7.92816(26)$ & 15.59 & 5.92 & 7.44 \\
510 & $35.407(6)$ & $17.7131(31)$ & $7.93036(28)$ & 18.21 & 6.39 & 7.96 \\
550 & $35.424(7)$ & $17.7206(34)$ & $7.93310(30)$ & 20.72 & 6.81 & 8.28 \\
600 & $35.442(7)$ & $17.730(4)$ & $7.93619(32)$ & 22.95 & 7.15 & 9.06
\end{tabular}

Rietveld refinement of PND data in space group $\mathrm{Bbm} 2$ was carried out for all data sets collected. Refined lattice parameters at each temperature are given in Table S1. These values are that of the supercell, c.f. Figure 3(c) within the main text in which lattice parameters are reduced to the aristotype cell.

The quality of the refinement shows a marked deterioration at approximately $470 \mathrm{~K}$ (as indicated by the increase in the goodness-of-fit parameters, Figure S2) corresponding to the loss of reflections associated with the expanded TTB cell. The data points at approximately $300 \mathrm{~K}$ represent the change in sample environment at ambient temperature. 


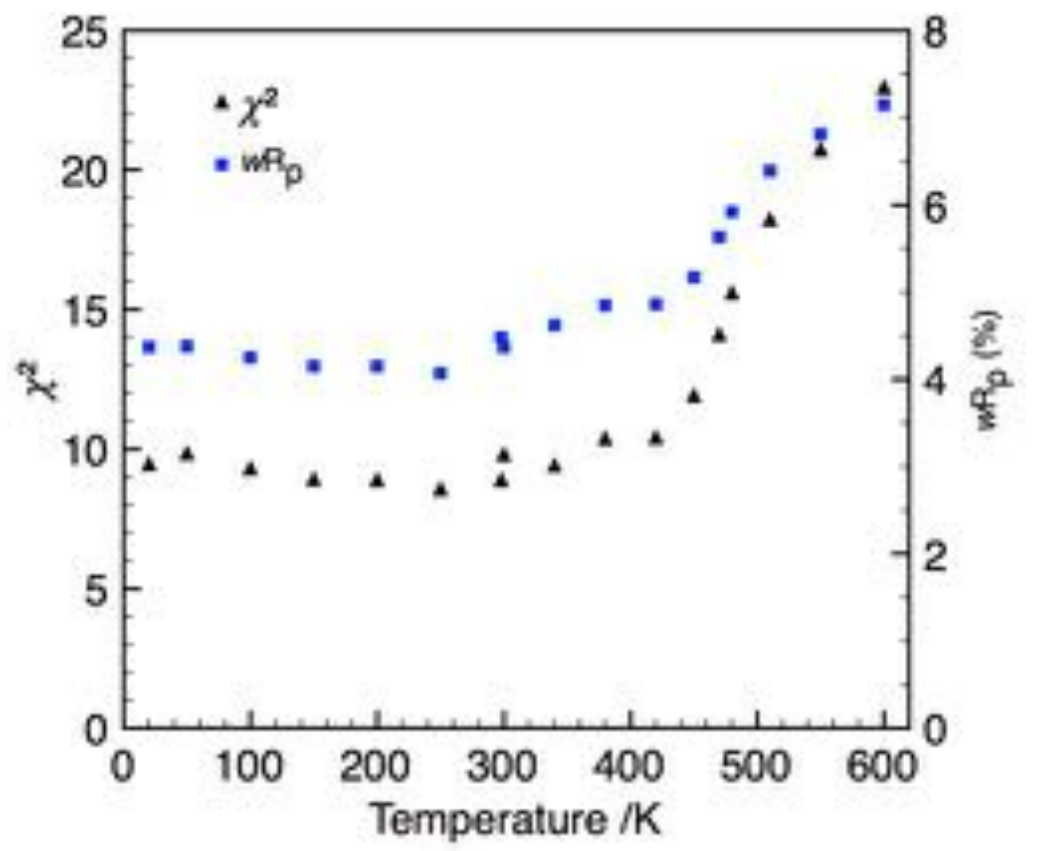

Figure S2. Refinement quality parameters; PND data for $\mathrm{Ba}_{4} \mathrm{La}_{0.67} \mathrm{Nb}_{10} \mathrm{O}_{30}(x=0)$ refined using space group Bbm2.

As discussed above, it was not possible to refine individual isotropic displacement parameters. When values for each site type (e.g. A1-site) were constrained, Table S2, a gradual increase is observed as expected. 
Table S3: Refined isotropic displacement parameters

\begin{tabular}{ccccc}
\hline Temp / K & \multicolumn{4}{c}{$\boldsymbol{\mu}_{\text {iso }} / \AA^{3}$} \\
& $\mathrm{Ba}$ & $\mathrm{La}$ & $\mathrm{Nb}$ & $\mathrm{O}$ \\
\hline 20 & $0.0081(11)$ & $0.0038(22)$ & $0.0048(4)$ & $0.0050(4)$ \\
50 & $0.0091(12)$ & $0.0053(23)$ & $0.0049(4)$ & $0.0052(4)$ \\
100 & $0.0088(10)$ & $0.0074(21)$ & $0.00571(33)$ & $0.00549(22)$ \\
150 & $0.0108(11)$ & $0.0060(22)$ & $0.0058(4)$ & $0.0058(4)$ \\
200 & $0.013(12)$ & $0.0070(22)$ & $0.0066(4)$ & $0.0063(4)$ \\
250 & $0.0154(12)$ & $0.0085(22)$ & $0.0075(4)$ & $0.0068(4)$ \\
300 & $0.0143(13)$ & $0.0115(25)$ & $0.0086(5)$ & $0.0078(4)$ \\
298 & $0.0158(13)$ & $0.0127(26)$ & $0.0102(5)$ & $0.0093(4)$ \\
340 & $0.0169(14)$ & $0.0136(27)$ & $0.0108(5)$ & $0.0102(4)$ \\
380 & $0.0177(15)$ & $0.0130(28)$ & $0.0109(5)$ & $0.0104(5)$ \\
420 & $0.0204(16)$ & $0.0136(28)$ & $0.0108(5)$ & $0.0104(5)$ \\
450 & $0.0234(17)$ & $0.0157(31)$ & $0.0119(6)$ & $0.0121(5)$ \\
470 & $0.0224(19)$ & $0.0147(33)$ & $0.0114(6)$ & $0.0119(6)$ \\
480 & $0.0243(22)$ & $0.014(4)$ & $0.0112(7)$ & $0.0126(7)$ \\
510 & $0.0243(22)$ & $0.014(4)$ & $0.0112(7)$ & $0.0126(7)$ \\
550 & $0.0262(24)$ & $0.013(4)$ & $0.0114(8)$ & $0.0129(7)$ \\
600 & $0.0283(26)$ & $0.016(4)$ & $0.0121(8)$ & $0.0138(8)$ \\
& & & &
\end{tabular}


Above $480 \mathrm{~K}$ the structure appears to have similar metrics to the basic high symmetry tetragonal aristotype (i.e. no reflections due to an extended superstructure are observed), however, based on synchrotron-PXRD a very slight orthorhombic distortion persists. A high temperature non-polar structure has been reported for other TTBs. The Rietveld refinement of PND data collected at $600 \mathrm{~K}$ with the orthorhombic non-polar space group Pbam is shown in figure S3. $\chi^{2}=4.471, w \mathrm{R}_{\mathrm{p}}=3.19 \%$
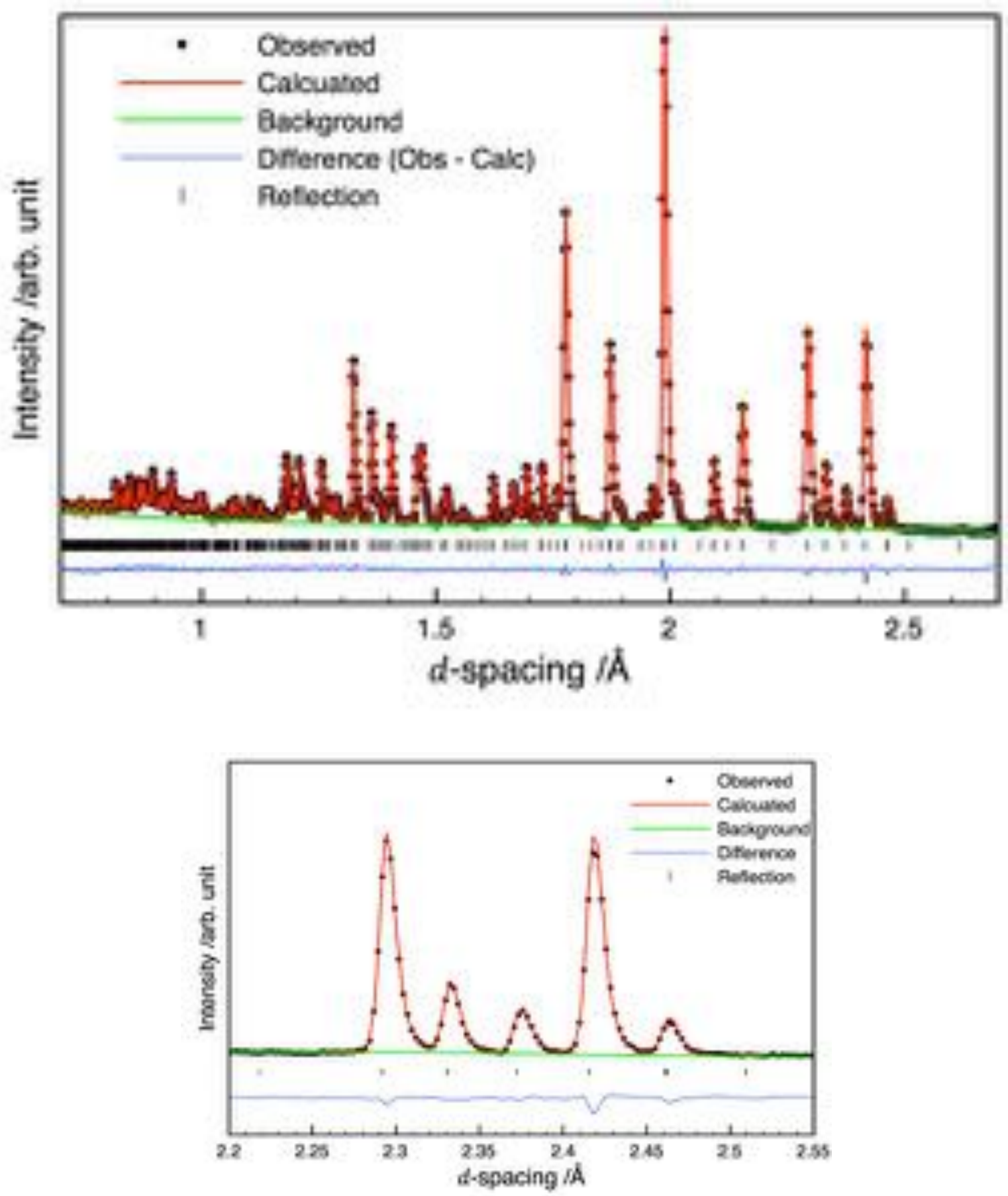

Figure S3. (a) Rietveld refinement of PND data for $\mathrm{Ba}_{4}\left(\mathrm{La}_{1-\mathrm{x}} \mathrm{Nd}_{\mathrm{x}}\right)_{0.67} \square_{1.33} \mathrm{Nb}_{10} \mathrm{O}_{30}, x=0$ (La) at $600 \mathrm{~K}$ in space group Pbam; (b) enlarged view of $d=2.2-2.55$ demonstrating lack of superstructure reflections. 
Synchrotron-PXRD data was collected on cooling from $500 \mathrm{~K}$ to $100 \mathrm{~K}$ for $\mathrm{Ba}_{4} \mathrm{La}_{0.67} \mathrm{Nb}_{10} \mathrm{O}_{30}$ $(x=0)$ on beamline I1 1 at the Diamond Light Source. A Rietveld refinement of data collected at $100 \mathrm{~K}$ using the same $B b m 2$ structural model is shown in Figure $\mathrm{S} 4\left(\chi^{2}=6.258, w \mathrm{R}_{\mathrm{p}}=\right.$ $11.13 \%)$. Figure S5 depicts enlarged sections of the same refinement. As with neutron data, individual atom positions and $\mu_{\text {iso }}$ were not refined.

The deficiencies in the refinement are principally from peak shape and intensity. Refinement of the positions of atoms in the $\mathrm{A} 2$ site $\left(\mathrm{Ba}^{2+}\right)$ and no others (data not shown) led to improvements of the fit $\left(\chi^{2}=5.690, w \mathrm{R}_{\mathrm{p}}=9.58 \%\right)$ indicating that while atom positions may be similar to the model described in the main text and which is based on the $\mathrm{Ba}_{4} \mathrm{Na}_{2} \mathrm{Nb}_{10} \mathrm{O}_{30}$ structural model of Labbé (Ref 25 in the main text), it is not identical.

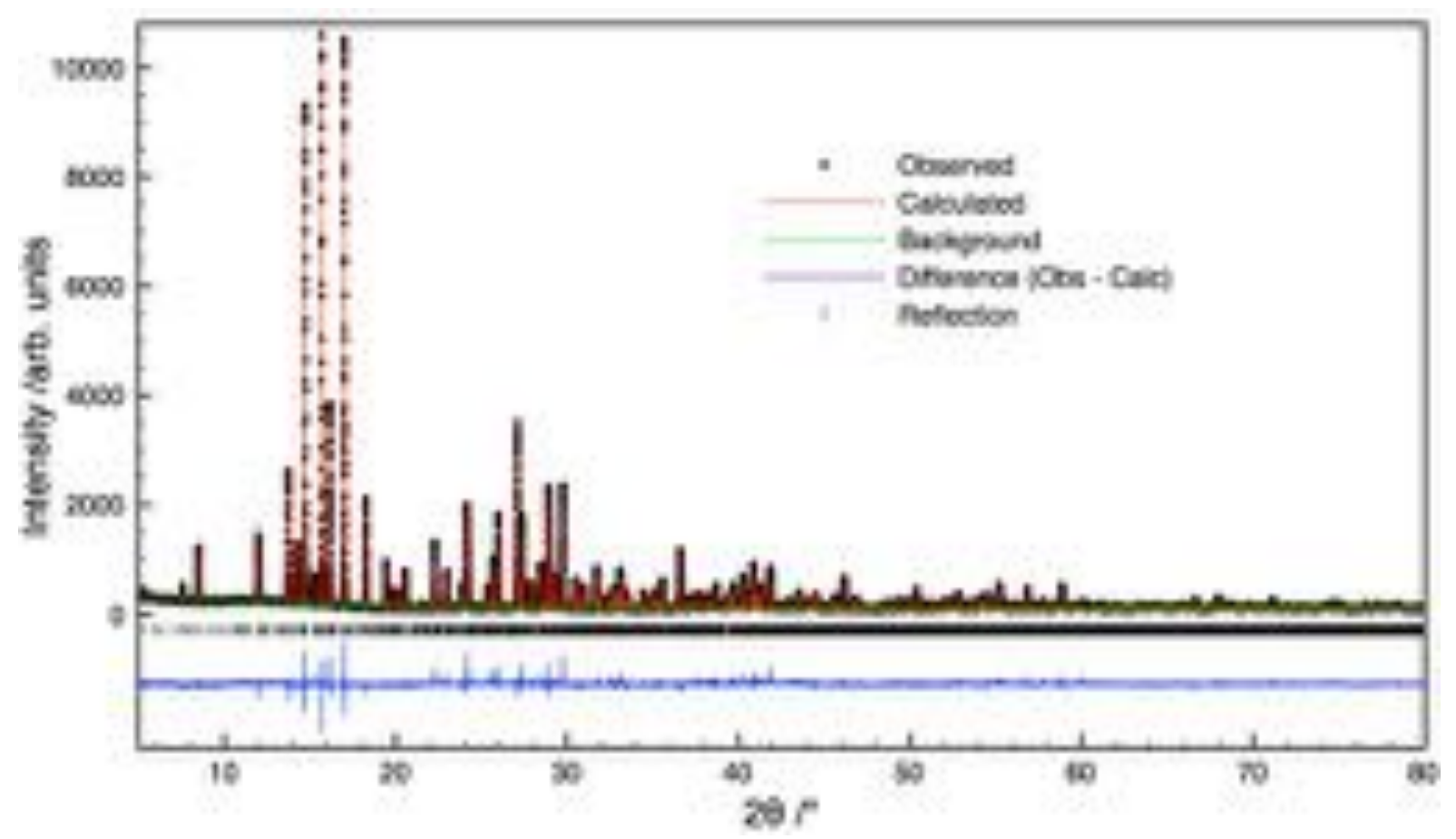

Figure S4. Rietveld refinement of s-PXRD data for $\mathrm{Ba}_{4}\left(\mathrm{La}_{1-\mathrm{x}} \mathrm{Nd}_{\mathrm{x}}\right)_{0.67} \square_{1.33} \mathrm{Nb}_{10} \mathrm{O}_{30}, x=0$ (La) at $100 \mathrm{~K}$ in space group Bbm2. 

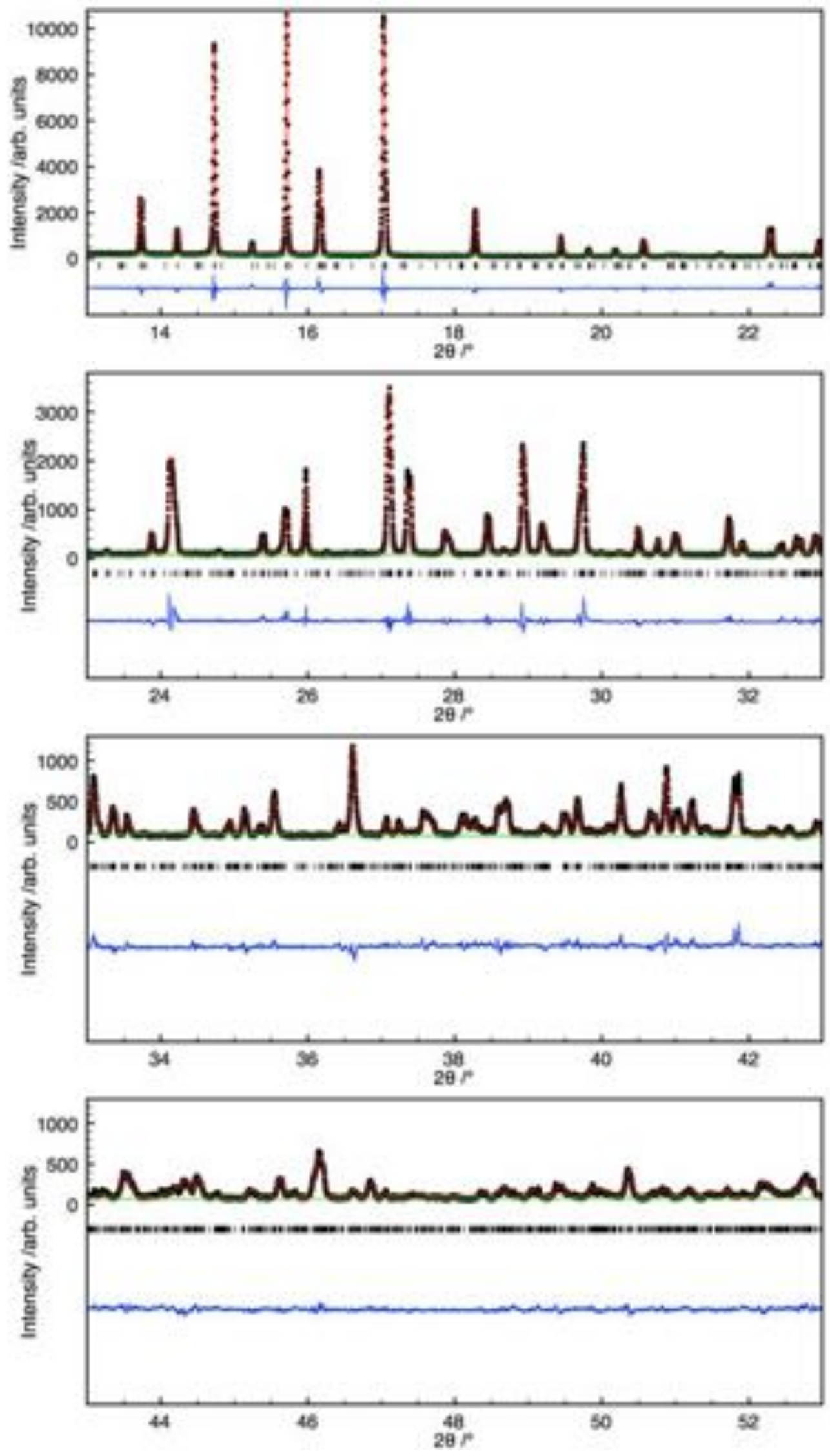

Figure S5. Enlarged sections of Figure S4 - s-PXRD data for $\mathrm{Ba}_{4}\left(\mathrm{La}_{1-\mathrm{x}} \mathrm{Nd}_{\mathrm{x}}\right)_{0.67} \square_{1.33} \mathrm{Nb}_{10} \mathrm{O}_{30}$; $x=0(\mathrm{La})$ at $100 \mathrm{~K}$ in space group $\mathrm{Bbm} 2$. 
Thermal expansion

Synchrotron PXRD data of $\mathrm{Ba}_{4} \mathrm{La}_{0.67} \mathrm{Nb}_{10} \mathrm{O}_{30}(x=0)$ indicates two regimes of linear thermal expansion at low and high temperature, Figure 3(c). The thermal expansion coefficient, $\alpha$, for individual cell parameters was calculated. Values obtained are as expected for an oxide material. Thermal expansion rate is greater in the higher temperature region (above $270 \mathrm{~K}$ ) and is larger in $a$.

Table S4. Thermal expansion coefficients for $\mathrm{Ba}_{4} \mathrm{La}_{0.67} \mathrm{Nb}_{10} \mathrm{O}_{30}(x=0)$ as determined form sPXRD data.

\begin{tabular}{|c|c|c|}
\hline \multirow{2}{*}{ Axis direction } & \multicolumn{2}{|c|}{$\begin{array}{c}\text { Linear thermal expansion coefficients, } \alpha_{L}\left(10^{-6} \mathrm{~K}^{-1}\right) \\
\text { Low T }(<270 \mathrm{~K})\end{array}$} \\
\hline $\boldsymbol{a}$ & 6.78 & High T $(>270 \mathrm{~K})$ \\
\hline $\boldsymbol{b}$ & 5.49 & 11.3 \\
\hline $\boldsymbol{c}$ & 2.56 & 7.64 \\
\hline
\end{tabular}




\section{Dielectric properties}

Dielectric data for the intermediate compositions, Figure S6, demonstrates the decreasingly diffuse nature of the low temperature peak with increasing $x$ (decreasing A1-cation size). The data correlate with the observed $P-E$ behaviour (Figures S8-10) which indicate a crossover from relaxor ferroelectric (RFE) to more 'normal' ferroelectric-like behaviour as the two peaks coalescence.

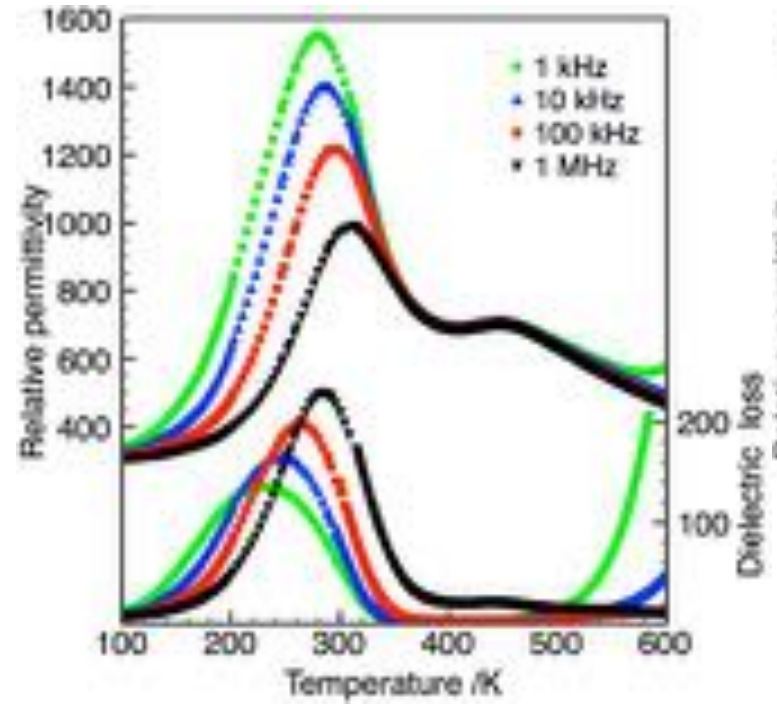

c) b)

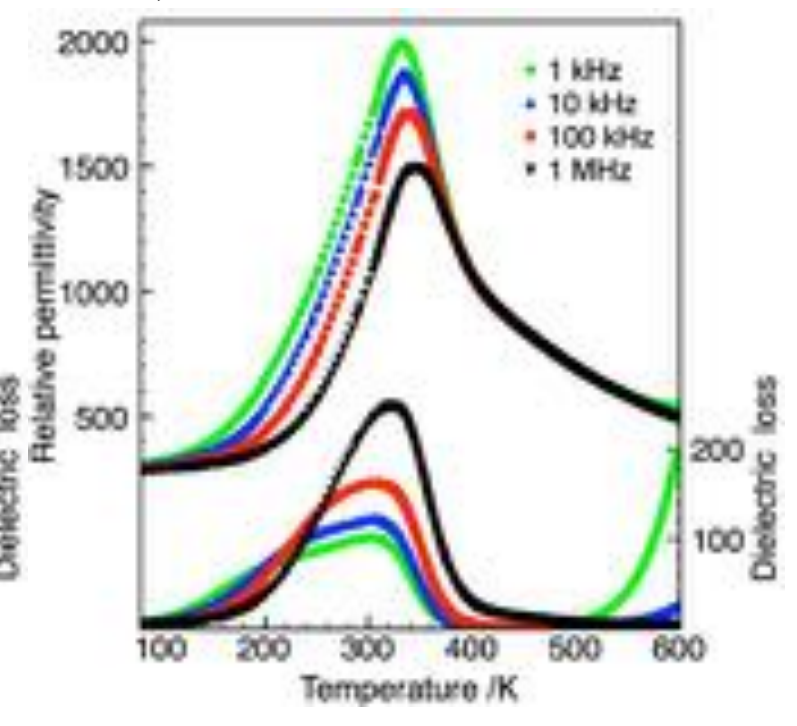

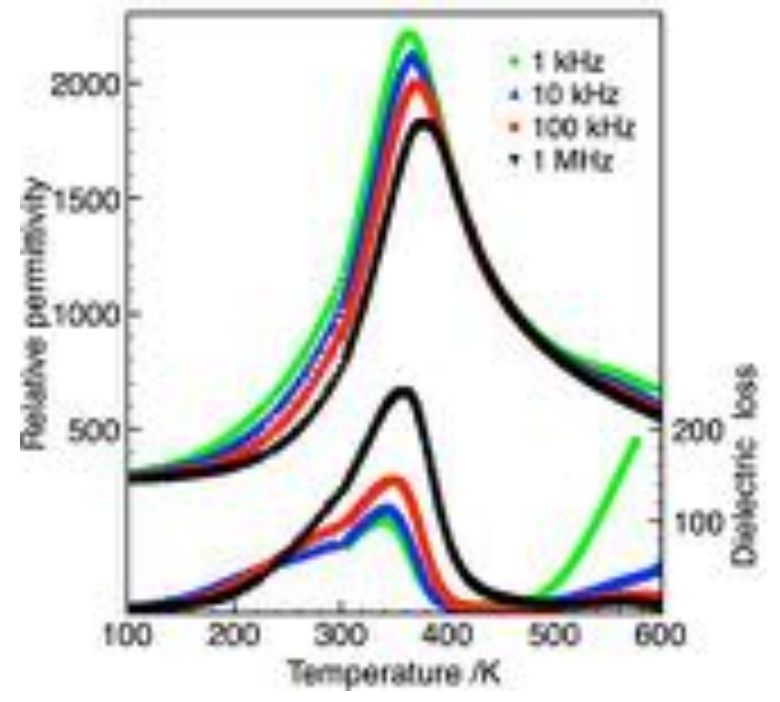

Figure S6. Relative permittivity $\left(\varepsilon^{\prime}\right)$ and dielectric loss $\left(\varepsilon^{\prime \prime}\right)$ for $\mathrm{Ba}_{4}\left(\mathrm{La}_{1-\mathrm{x}} \mathrm{Nd}_{\mathrm{x}}\right)_{0.67} \mathrm{Nb}_{10} \mathrm{O}_{30}$ at selected frequencies for: (a) $x=0.25$, (b) $x=0.5$ and (c) $x=0.75$ 
With increasing $x$, the contribution of the high temperature relaxation is shown to become more influential, Figure S7.
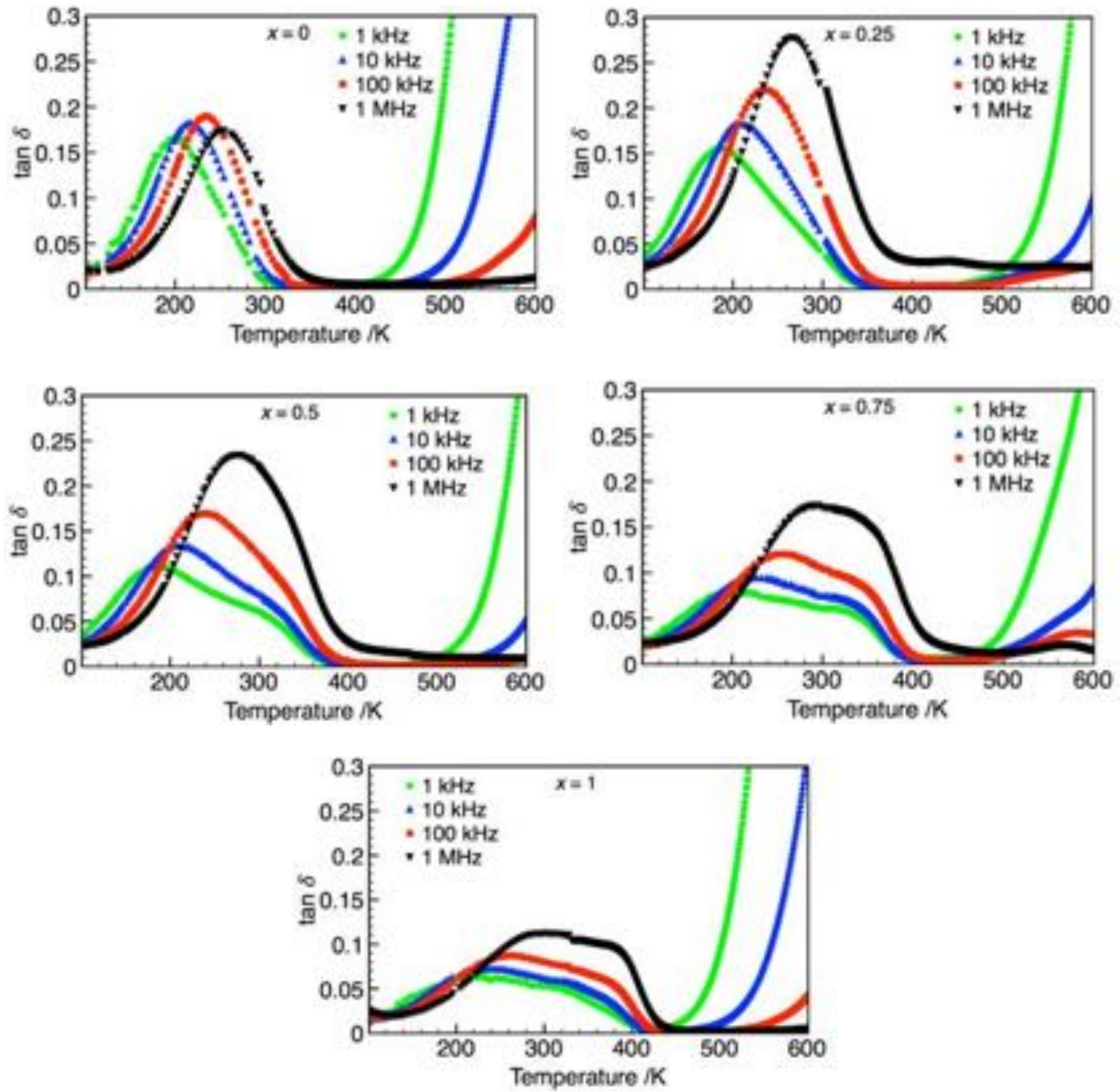

Figure S7. Dielectric loss, $\tan \delta$, for $\mathrm{Ba}_{4}\left(\mathrm{La}_{1-\mathrm{x}} \mathrm{Nd}_{\mathrm{x}}\right)_{0.67} \mathrm{Nb}_{10} \mathrm{O}_{30}$ at selected frequencies for $x=$ $0,0.25,0.5,0.75,1$. 


\section{Polarisation-Field (P-E) measurements for $\mathrm{Ba}_{4}\left(\mathrm{La}_{1-\mathrm{x}} \mathrm{Nd}_{\mathrm{x}}\right)_{0.67} \mathrm{Nb}_{10} \mathrm{O}_{30}$}

Polarisation $v s$. field measurements for $x=0$ (Figure S8), $x=0.25$ (Figure S9), $x=0.5$ (Figure S10) and $x=0.75$ (Figure S11). All compounds are ferroelectric at low temperature; wellsaturated ferroelectric hysteresis loops are observed which become slimmer with increasing temperature. As with $\mathrm{Ba}_{4} \mathrm{La}_{0.67} \mathrm{Nb}_{10} \mathrm{O}_{30}(x=0)$ P-E loops with 'pinched' character are recorded in the temperature range between the two peaks in the dielectric data shown for $x=$ 0.25 . No indication of the onset of such pinched loops is observed for $x=0.75$ or $x=1$; for these compositions the saturated P-E loop becomes slimmer and at high temperature a linear dielectric response indicates loss of ferroelectricity.
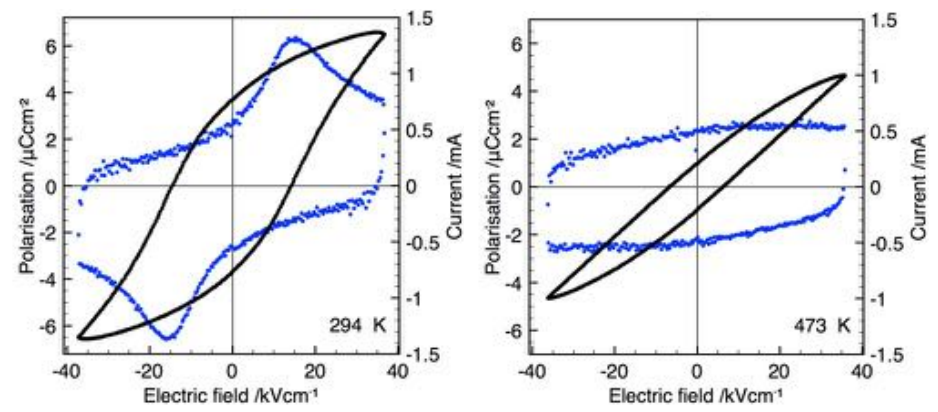

Figure S8. P-E data for $\mathrm{Ba}_{4}\left(\mathrm{La}_{1-\mathrm{x}} \mathrm{Nd}_{\mathrm{x}}\right)_{0.67} \mathrm{Nb}_{10} \mathrm{O}_{30}, x=1$, obtained at $100 \mathrm{~Hz}$.
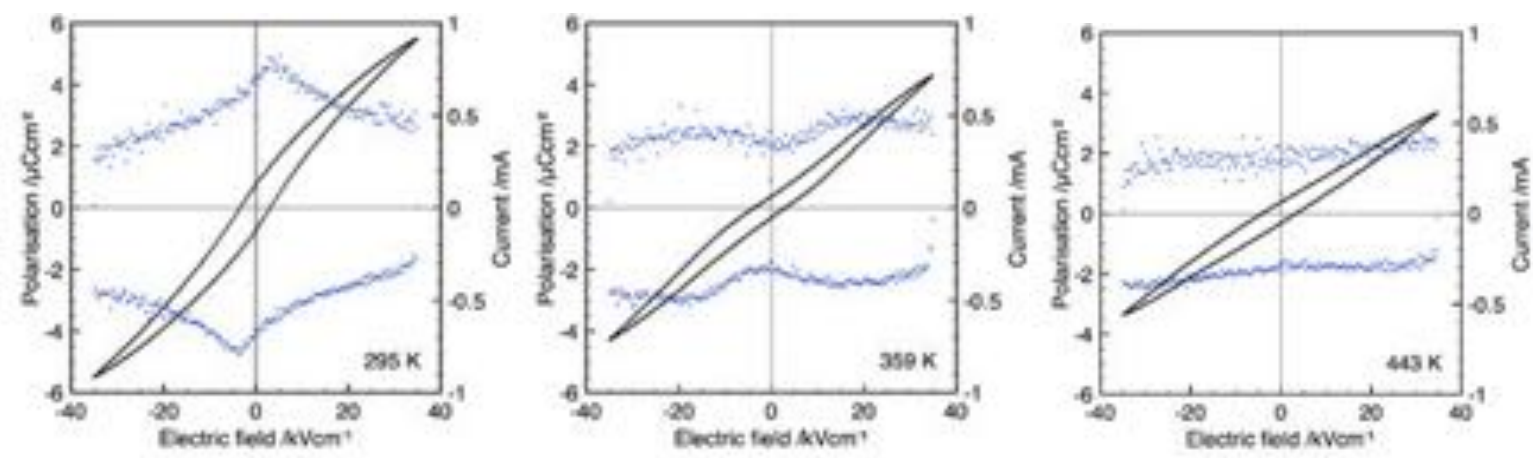

Figure S9. Variable temperature P-E data for $\mathrm{Ba}_{4}\left(\mathrm{La}_{1-\mathrm{x}} \mathrm{Nd}_{\mathrm{x}}\right)_{0.67} \mathrm{Nb}_{10} \mathrm{O}_{30}, x=0.25(100 \mathrm{~Hz})$.
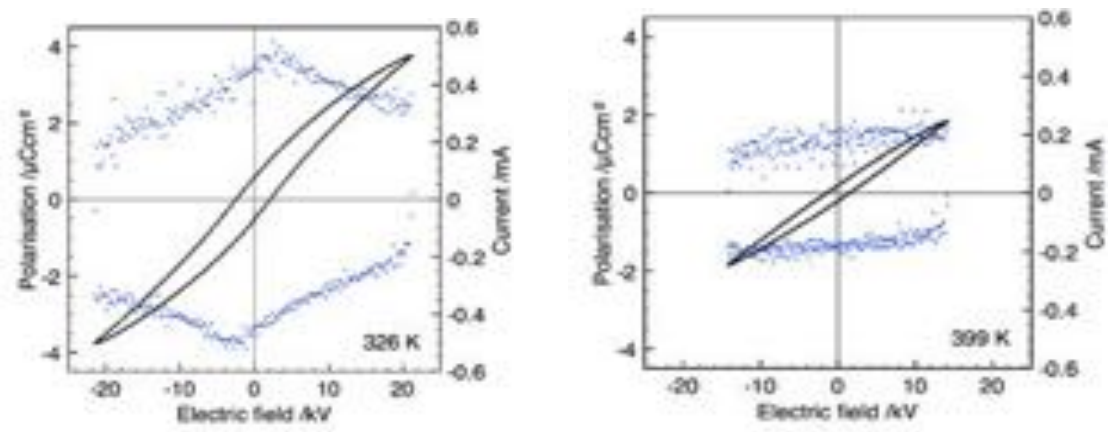

Figure S10. P-E data for $x=0.5(100 \mathrm{~Hz})$ 

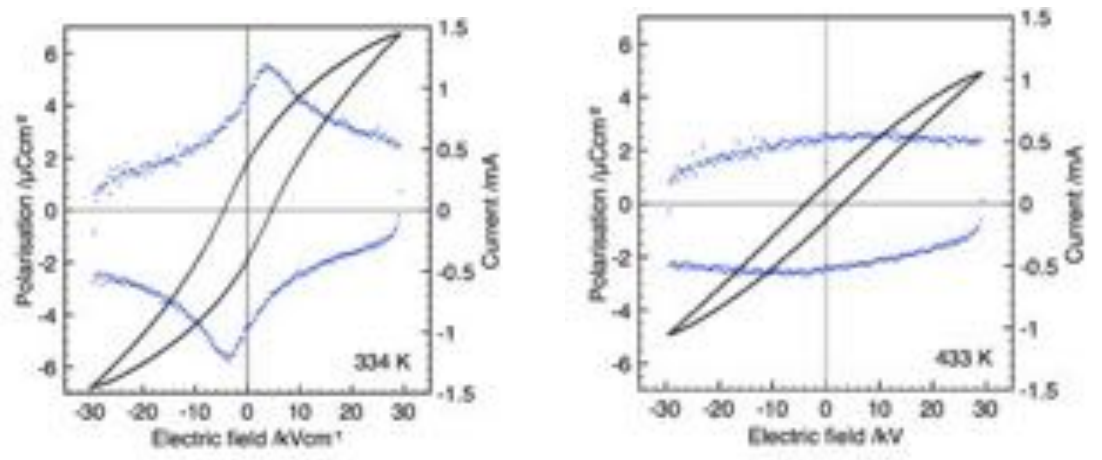

Figure S11. P-E data for $x=0.75(100 \mathrm{~Hz})$ 


\section{Quenching}

Samples of $\mathrm{Ba}_{4} \mathrm{La}_{0.67} \mathrm{Nb}_{10} \mathrm{O}_{30}(x=0)$ were air quenched from high temperature.

Samples with sputtered Au electrodes were heated to $623 \mathrm{~K}\left(350^{\circ} \mathrm{C}\right)$ for 2 hours and short circuited while earthed. Samples were then air quenched and data collected immediately. 'Pinched' behaviour persists, Figure S12.
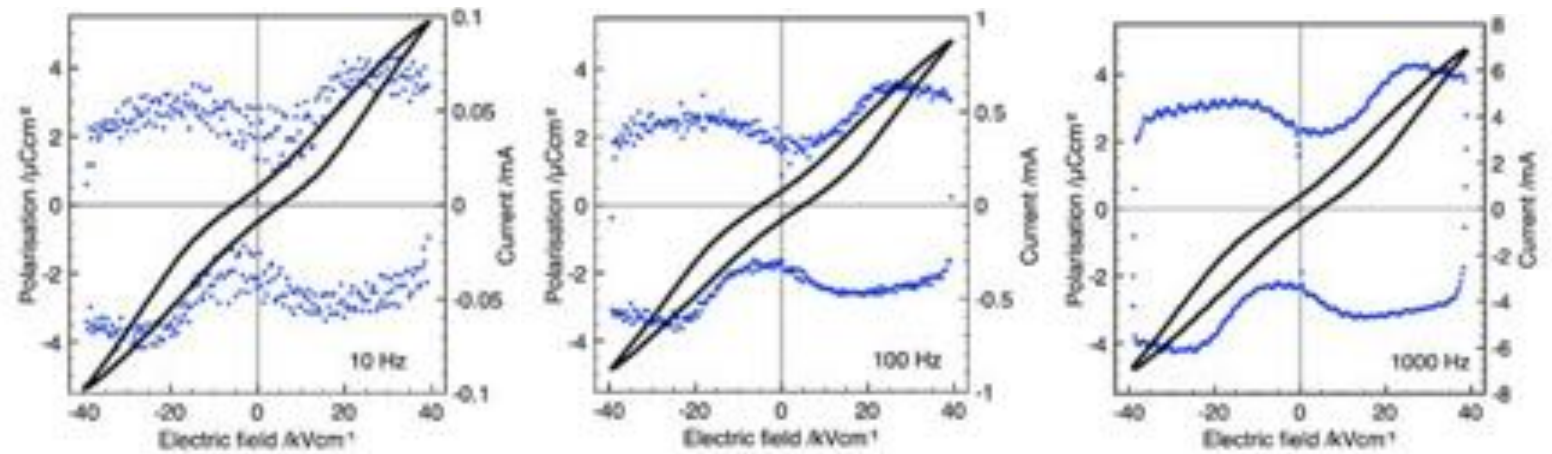

Figure S12. P-E data for sample quenched from $623 \mathrm{~K}$ at $10 \mathrm{~Hz}, 100 \mathrm{~Hz}$ and $1000 \mathrm{~Hz}$ demonstrating retention of pinched character of P-E loop, (295 K).

Higher temperature quenching experiments were also carried out; samples were heated to $1273 \mathrm{~K}\left(1000{ }^{\circ} \mathrm{C}\right)$ for 2 hours and air quenched. Sputtered electrodes may degrade at such temperatures so silver conductive paint was applied as soon as samples were cool enough and data recorded (within 30 minutes of quenching). Again 'pinched' behaviour persists, Figure S13.

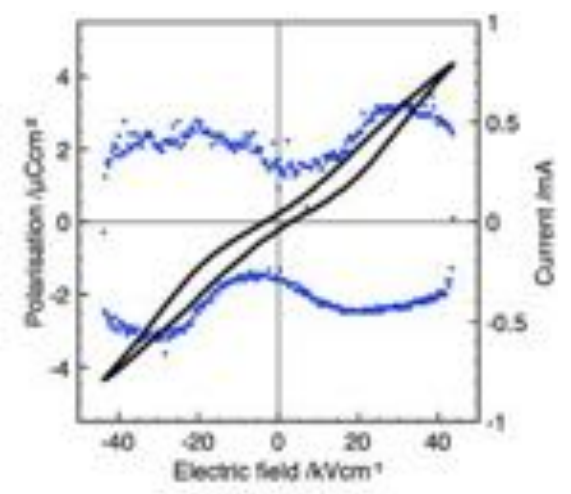

Figure S13. P-E data for sample quenched from $1273 \mathrm{~K}$ demonstrating retention of the pinched character of $\mathrm{P}-\mathrm{E}$ loop, $100 \mathrm{~Hz}$, ambient temperature $(295 \mathrm{~K})$. 


\section{Fatigue measurements}

A sample of $\mathrm{Ba}_{4} \mathrm{La}_{0.67} \mathrm{Nb}_{10} \mathrm{O}_{30}(x=0)$ was fatigued for $1 \times 10^{7}$ cycles with an applied electric field of $40 \mathrm{kVcm}^{-1}$ (fatigue frequency $=10 \mathrm{kHz}$ ) at ambient temperature (296 K). P-E data (applied field $40 \mathrm{kVcm}^{-1}, 100 \mathrm{~Hz}$ ) was recorded at specific points during the fatiguing process. There is little appreciable difference between the first and last loop, Figure S14.

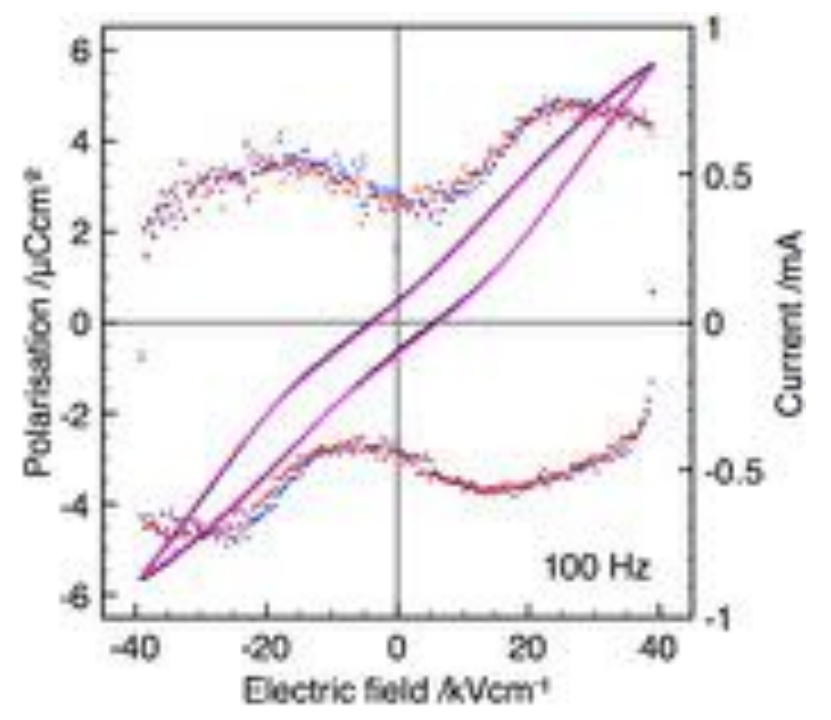

Figure S14. Comparison of $1^{\text {st }}$ (polarisation data in black, current data in blue) and last $(1 \times$ $10^{7}$ ) cycle (polarisation data purple, current data red), recorded at ambient temperature.

The maximum polarisation $\left(\mathrm{P}_{\max }\right)$ and the remenant polarisation $\left(\mathrm{P}_{\mathrm{r}}\right)$ do not significantly change during the fatiguing process, Figure S15

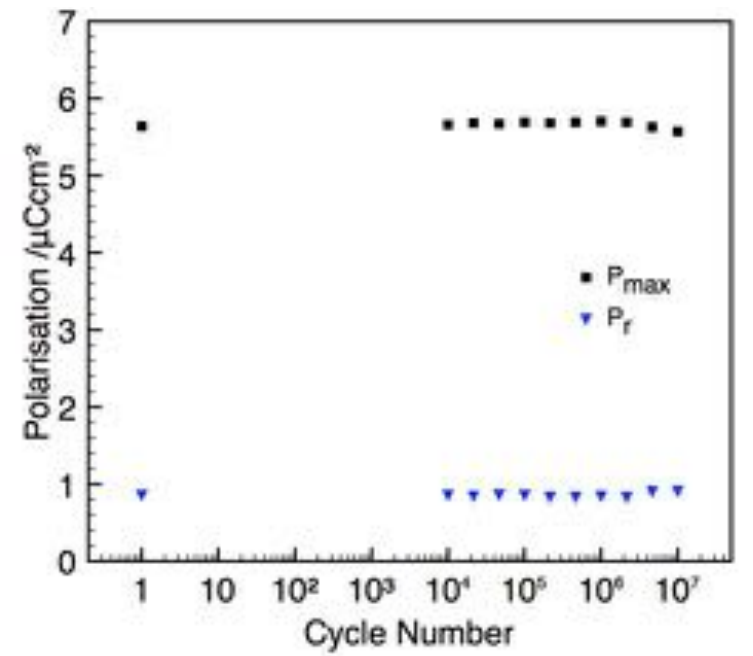

Figure S15. Maximum polarisation and remenant polarisation during fatiguing process. 